\title{
Improving Write Performance and Extending Endurance of Object-Based NAND Flash Devices
}

\author{
JIE GUO and CHUHAN MIN, University of Pittsburgh \\ TAO CAI, Jiangsu University \\ YIRAN CHEN, Duke University
}

\begin{abstract}
Write amplification is a major cause of performance and endurance degradations in NAND flash-based storage systems. In an object-based NAND flash device (ONFD), two causes of write amplification are onode partial update and cascading update. Here, onode is a type of small-sized object metadata, and multiple onodes are stored in one NAND flash page. Updating one onode invokes partial page update (i.e., onode partial update), incurring unnecessary migration of the un-updated data. Cascading update denotes updating object metadata in a cascading manner due to object data update or migration. Although there are only several bytes that need to be updated in the object metadata, one or more pages have to be re-written accordingly. In this work, we propose a system design to alleviate the write amplification issue in the object-based NAND flash device. The proposed design includes (1) a multi-level garbage collection technique to minimize unnecessary data migration incurred by onode partial update and (2) a B + table tree, Semantics-Aware Flexible (SAF) data layout, and selective cache design to reduce the write operations associated with cascading update. To guarantee system consistency, we also propose a power failure handling technique. Experiment results show that our proposed design can achieve up to $20 \%$ write reduction compared to the best states of the art.
\end{abstract}

CCS Concepts: $\bullet$ Information systems $\rightarrow$ Flash memory; $\bullet$ Hardware $\rightarrow$ Non-volatile memory; $\bullet$ Software and its engineering $\rightarrow$ Secondary storage;

Additional Key Words and Phrases: NAND flash memories, write amplification, performance

\section{ACM Reference format:}

Jie Guo, Chuhan Min, Tao Cai, and Yiran Chen. 2017. Improving Write Performance and Extending Endurance of Object-Based NAND Flash Devices. ACM Trans. Embed. Comput. Syst. 17, 1, Article 18 (November 2017), 26 pages.

https://doi.org/10.1145/3105924

\section{INTRODUCTION}

Thanks to the advantages in fabrication cost and non-volatility, NAND flash memory has achieved remarkable success in modern storage systems ranging from low-power embedded systems to high-end servers. However, NAND flash memory is also known for an "erase-before-program" limitation; that is, an erase operation must be performed before programming a NAND flash page. This erase-before-program (a.k.a. out-of-place update) property causes more data than requested

This work was supported by NSF CNS-1253424, CNS-1615475, and AFRL FA8750-15-1-0176.

Author addresses: J. Guo and C. Min, Department of Electrical and Computer Engineering, University of Pittsburgh, Pittsburgh, PA, USA 15213; emails: \{jig26, chm114\}@pitt.edu; T. Cai, Department of Computer Science and Engineering, Jiangsu University, Jiangsu, China, 212013; email: caitao@ujs.edu.cn; Y. Chen, Department of Electrical and Computer Engineering, Duke University, Durham, NC, USA 27708; email: yiran.chen@duke.edu.

Permission to make digital or hard copies of all or part of this work for personal or classroom use is granted without fee provided that copies are not made or distributed for profit or commercial advantage and that copies bear this notice and the full citation on the first page. Copyrights for components of this work owned by others than ACM must be honored. Abstracting with credit is permitted. To copy otherwise, or republish, to post on servers or to redistribute to lists, requires prior specific permission and/or a fee. Request permissions from permissions@acm.org.

(C) 2017 ACM 1539-9087/2017/11-ART18 \$15.00

https://doi.org/10.1145/3105924

ACM Transactions on Embedded Computing Systems, Vol. 17, No. 1, Article 18. Publication date: November 2017. 


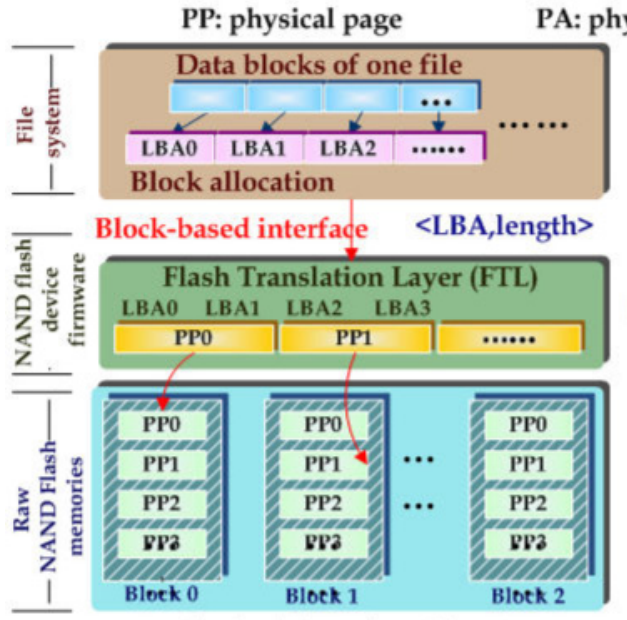

(a) Block-based model

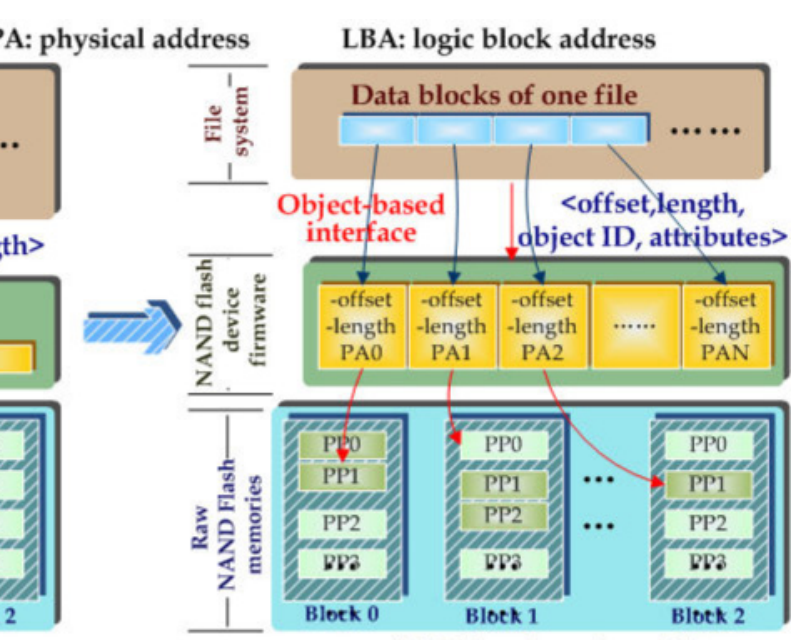

(b) Object-based model

Fig. 1. The block-based storage vs. the object-based storage.

to be programmed during garbage collection, that is, write amplification (Lu et al. 2013a; Hu et al. 2009; Guo et al. 2013a). Since the program operation has long latency and determines system endurance together with erase operation, a direct result of write amplification is degradations of system performance and endurance. With technology node scaling down, both write performance and endurance of NAND flash memory continuously degrade: The programming latency increases to $3 \mathrm{~ms}$ (Zhao et al. 2013) at $2 \mathrm{Xnm}$ technology node while the rated memory endurance under the $1 \mathrm{Xnm}$ technology node decreases to 3,000 P/E cycles (Yoon and Tressler 2012). Minimizing write amplification becomes critical to enhance the performance and reliability of NAND flash-based storage system.

One way to minimize write amplification is to reduce data migration of garbage collection. This goal can be achieved by optimizing the layout of hot and cold data (Hsieh et al. 2006; Lee et al. 2008). Unfortunately, the existing block-based storage model cannot accurately differentiate hot and cold data. As shown in Figure 1(a), in the block-based model, the logic block is the basic data storage unit. The file system divides the storage space of the NAND flash device into a number of logic blocks. Files data are divided into a number of data blocks and stored in these logic blocks. The data block with its logic block address (LBA) and data length is sent to the NAND flash device through block-based interface. Logic block allocation is handled by storage management layer of file system. Being unaware of block allocation policies (Chidambaram et al. 2012) and file semantics, the underlying NAND flash device cannot accurately identify data access patterns. Moreover, to accommodate the out-of-place property, an indirection table is deployed in the NAND flash device to map logic block address (LBA) to physical address. The size of logic block is only 512 bytes, less than the size of a NAND physical page. FTL groups $N$ consecutive logic blocks in one logic page and stores the data belonging to one logic page to one physical page. In the example of Figure 1(b), $N$ is equal to 2. The indirection table maintains mapping between the logic page address and the physical page address, significantly raising memory consumption (Lu et al. 2013b; Gupta et al. 2008). The indirection table can also incur high performance overhead when coarser mapping granularity is adopted (Lee et al. 2008). To overcome the architectural limitation of the block-based storage model, an object-based storage model was proposed (Rajimwale et al. 2009). As shown in Figure 1(b), the object-based model manages data in unit of object (e.g., a file in the 
object file system). The file data blocks, the data length, and the offset within the file are sent to the the object-based NAND flash device (ONFD) through an object-based interface (Rajimwale et al. 2009; Kang et al. 2011). With object semantics, the ONFD can improve the accuracy of data pattern identification (Kang et al. 2011; Wang et al. 2014b). As a result, garbage collection induced data migration can be reduced. In addition, by offloading the object storage management to the ONFD, the indirection table is removed from the ONFD, significantly simplifying the system design and reducing memory consumption.

In this article, we continuously explore the system optimization space in the ONFD by leveraging object semantics. Two causes of write amplification in the ONFD are identified. The first one is onode partial update. Onode is a data structure in the ONFD to store object attributes (Kang et al. 2011). Due to its small size, multiple onodes are stored in one physical page to reduce internal fragmentation (Kang et al. 2011). Updating one of the onodes incurs partial update of a physical page, which is called onode partial update. Since the write is performed in unit of page in the ONFD (Lu et al. 2013a), onode partial update invokes unnecessary migration of the un-updated data. Another cause of write amplification is cascading update. In the ONFD, physical addresses of object data are maintained in the per-object index. The per-object index is implemented with an extent B+ tree (Lee et al. 2008). The physical address of the per-object index root node page is stored in the onode (Kang et al. 2011). Due to out-of-place update property, updating physical address of object data causes the per-object index node pages to be updated in a cascade manner (Kang et al. 2007). Moreover, the existing design stores onode and per-object index root node separately. The new per-object index root node page address has to be updated in the onode, incurring more page write operations. Although onode partial update and cascade update only overwrites several bytes, more than one page has to migrate entirely, incurring significant write amplification.

To reduce the write amplification in the ONFD, we propose a system design to minimize the data migration incurred by onode partial update and cascading update. Our technical contributions can be summarized as follows:

- We propose a multi-level garbage collection (MLGC) technique to reduce the write operations induced by onode partial update. By grouping multiple onode partial updates and moving the remaining valid data at once, MLGC can significantly reduce the amount of migrating data.

- We propose a Semantics-Aware Flexible (SAF) data layout to reduce cascade update. By storing small-sized object data or per-object index and attributes within one physical page, migration of the un-updated data can be reduced.

- We propose a $\mathrm{B}+$ table tree to reduce cascading update. The $\mathrm{B}+$ table tree transfers address update of a node page from its parent node to a page table with virtual addresses. By optimizing the structure of the page table, we can effectively reduce the update to per-object indices.

- We propose a selective cache design to further minimize cascading update. The selective cache reduces write operations by merging object updates. To maximize the cache utilization, a data-type-dependent caching strategy and a node-dependent cache replacement policy are proposed.

- The information of invalid bytes of MLGC, the page table of B+table tree and the selective cache are all stored in DRAM, which exposes the system to the risk of inconsistency during power failure. To restore the system consistency, we also propose a power failure handling technique.

We evaluate the efficiency of our proposed design under five workloads that represent various applications. The experimental results show that compared with the best state-of-the-art works, 


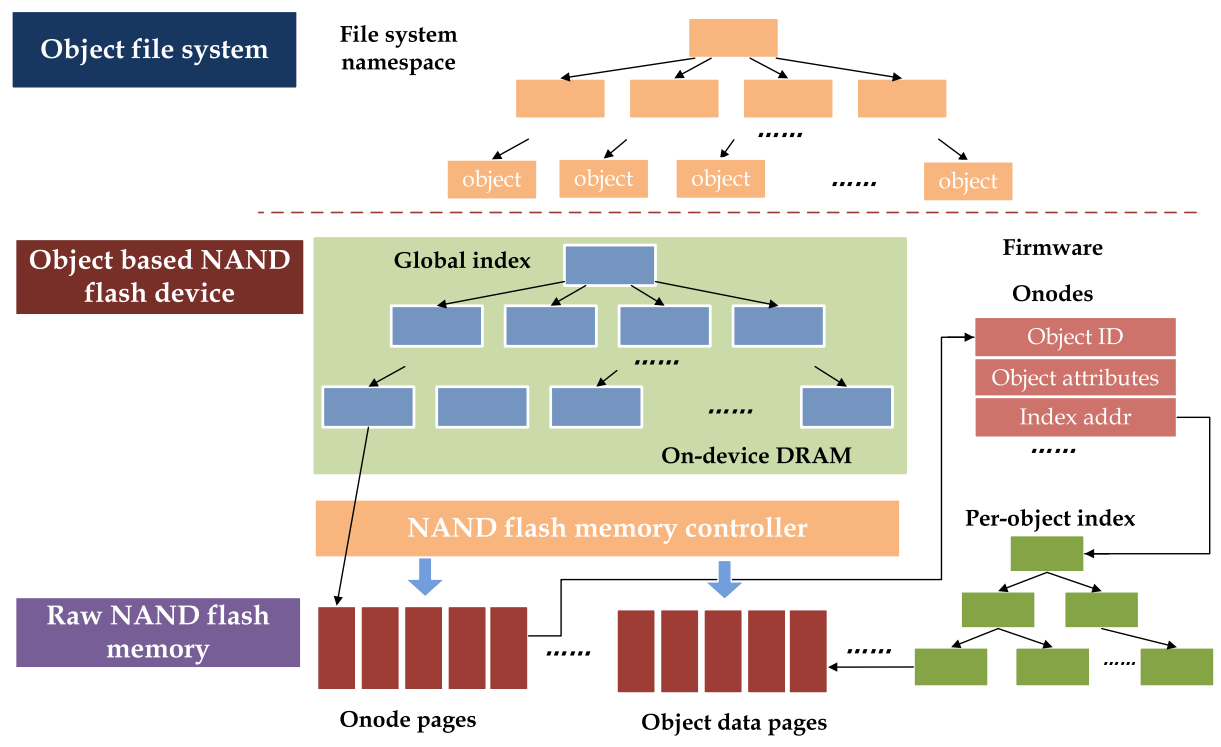

Fig. 2. The architecture of object-based storage system.

the proposed design can achieve up to $20 \%$ performance improvement and extend system lifetime by $76 \%$. The remainder of our article is organized as follows: Section 2 introduces the background knowledge; Section 3 presents the research motivation; Section 4 to Section 8 describe MLGC, SAF data layout, B+ table tree, selective cache, and the power failure handling technique in detail, respectively; Section 9 presents the experimental results; Section 10 introduces the related works; and Section 11 concludes this work.

\section{BACKGROUND}

A NAND flash memory adopts a block-page structure: It is divided into a number of blocks, and each block is divided into a number of pages. Erase operation is conducted in unit of block while program operation is performed in unit of page. The page size ranges from 512B to $16 \mathrm{~KB}$ (Grupp et al. 2012; Wang et al. 2014a; Guo et al. 2015). A NAND flash memory page (i.e., a physical page) consists of a data area and an out-of-band (OOB) area. The data area stores page data and the OOB area stores page metadata such as page data information and Error correcting codes (ECC) parity (Lu et al. 2013a; Guo et al. 2017). In a write operation, both the page metadata and the page data are programmed together to a NAND flash page.

The object-based NAND flash storage model is shown in Figure 2. In this model, object serves as the basic storage unit. An object denotes a variable-sized data container, which is uniquely identified by an 64-bit object ID. Typically, an object represents a file as in our article. Each object includes data and attributes (Nagle et al. 2008). The object data contains variable-length file data. The object attributes are file metadata such as ownership and access control. The object-based model includes an object file system and an ONFD. The object file system only maintains the name space. The ONFD manages the storage of objects. In general, an ONFD consists of raw NAND flash memories, a NAND flash memory controller, an on-device DRAM, and firmware. The NAND flash memory controller provides a datapath and applies data protection, for example, ECC. The DRAM is deployed to buffer frequently accessed data (Guo et al. 2013b). The firmware handles object management and NAND flash memory management. 


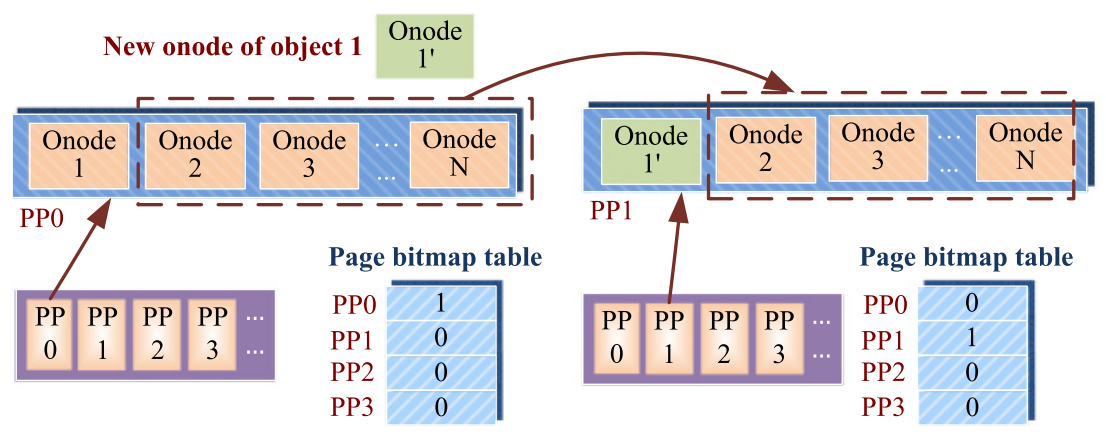

Fig. 3. An example of onode partial update.

In the ONFD, the object data are accessed via object metadata, which are also stored in NAND flash memories. The object metadata includes onodes and per-object indices. Each object has one onode, which contains the object attributes. The object attributes can have different length and therefore the onode also has a variable length. The onode also maintains the physical address of the per-object index root node page. The per-object index, with a tree structure, maintains the physical addresses of the object data. The physical addresses of the onodes are maintained in a global index. The global index, which is arranged with a B+ tree data structure, resides in the DRAM for fast access.

The ONFD also has the flash management functionality like physical page allocation, garbage collection, and wear-leveling. The ONFD adopts a log-structured page allocation method (Rosenblum and Ousterhout 1992). The physical storage space is divided into a number of chunks, each of which contains one or more NAND flash blocks. Garbage collection is performed in unit of chunk. Each chunk has a chunk metadata to maintain garbage collection information. The chunk metadata includes chunk states, valid page count, and page bitmap table (Lu et al. 2013a).

\section{MOTIVATION}

Two causes of write amplification in the ONFD are onode partial update and cascading update. In the ONFD, the onode size is usually smaller than the physical page size. To reduce internal fragmentation, more than one onode is stored in a physical page (Kang et al. 2011). Updating an onode causes partial page update, since the old onode is invalidated while the un-updated onodes in the same page still remain valid. The existing ONFD, however, only allows page-level invalidation with a page bitmap table at the granularity of page (Lu et al. 2013a). To conform to the page-level invalidation, the remaining valid bytes have to migrate. An example of onode partial update is shown in Figure 3. The physical page (PP) 0 stores the onodes of object $1 \sim \mathrm{N}$ (Onode $1 \sim$ Onode $\mathrm{N}$ ). The validity of PP0 data is maintained in the page bitmap table. When the new onode of object 1 (Onode $1^{\prime}$ ) is received, Onode 1 stored in PP0 is invalidated. Before invalidating PP0, the un-updated onodes (Onode $2 \sim$ Onode $\mathrm{N}$ ) must migrate to PP1 together with Onode $1^{\prime}$. Considering a typical onode size is $\sim 100$ bytes (Kang et al. 2011), for a physical page size of $2 \mathrm{~KB}$, the actual amount of data that are programmed is nearly $20 \times$ of the one to be updated. Even worse, with the increase of the physical page size, onode page update will aggravate the write amplification.

Another reason of write amplification in ONFD is cascading update. In ONFD, onode, per-object index and data belonging to the same object are stored in different physical pages (Kang et al. 2007). The per-object index is implemented with an extent B+ tree (Lee et al. 2008). The leaf node 

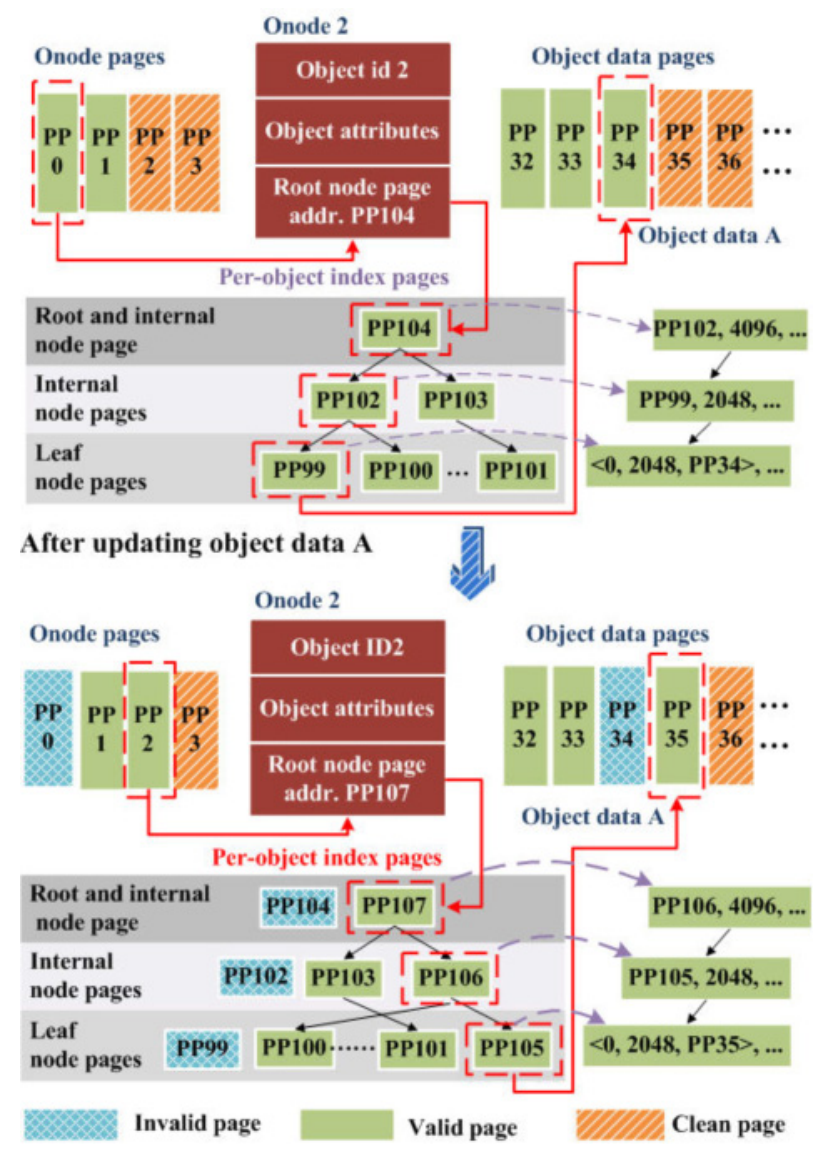

Fig. 4. An example of cascading update.

page records <offset, length, the physical addresses $>$ of the object data. The internal node page stores $<$ data offset, child node page address $>$ pairs, that is, key-value pairs. We call the extent $\mathrm{B}+$ tree recording the physical addresses of a child node page PA-extent $B+$ tree. When object data are updated or migrate during garbage collection, the new data address is updated in the leaf node page. Due to the out-of-place update property, updating a child node page causes its new address to be updated in the parent node page in a cascading manner until the root node page is reached (Kang et al. 2007). In addition, updating the address of the root node page also incurs onode update. Although there are only several bytes of the object metadata need to be updated, one page or more has to be migrated entirely, incurring significant write amplification. An example of a cascading update is shown in Figure 4. Object data A with offset 0 and length 2048 is stored in PP34. The physical address of object data $\mathrm{A}<0,2048, \mathrm{PP} 34>$ is recorded in the leaf node page PP99. When object data A migrates to PP35, updating the new address causes the leaf node page to be updated in PP105. Similarly, the new address of the leaf node page PP105 is updated in its parent node page, causing the parent node page to be updated in PP106. The cascading update continues until the root node page is updated to PP107, and the new root node page address is updated in the onode. In this case, overwriting one object data page incurs write operations of four object metadata pages. 
In addition to the intrinsic issue of binary-tree structure, a sub-optimal data layout also aggravates cascading update between per-object index and onode. The existing ONFD design employs data-separation-based data layout. Onode and the per-object index nodes are stored in different pages (Kang et al. 2007). Similarly, for the small objects with the data size smaller than one physical page, the corresponding onode and data are stored in different physical pages (Kang et al. 2007). Such a data separation causes at least two pages to be written on object data update and incurs a large amount of un-updated data to migrate.

\section{MULTI-LEVEL GARBAGE COLLECTION}

We propose MLGC to mitigate onode partial update incurred write amplification. MLGC employs a byte-state GC table. When an onode partial update occurs, instead of moving the valid bytes in the same page immediately, MLGC records the information of the invalid bytes <physical page number, offset, length $>$ in the byte-state GC table. Through grouping the invalid bytes incurred by more than one onode partial updates in a physical page and moving the remaining valid bytes at one time, data migration can be effectively reduced. However, the memory consumption of the byte-state GC table is prohibitively high if we record the invalid bytes information of every physical page. For example, in a 1TB ONFD with a $4 \mathrm{~KB}$ page size and a maximum $32 \mathrm{~GB}$ file size, each invalid bytes information entry can be represented with 12 bytes. The total size of the bytestate GC table is at least 3GB if we assume that each page has only one invalid bytes information entry.

To control memory consumption, we introduce two tables in MLGC for garbage collection: (1) a page bitmap table that records statuses of all physical pages and (2) limited-sized byte-state GC table that only records invalid bytes information entries of some physical pages. The byte-state GC table is implemented with a hash table and single link tables. The hash table is used to fast locate the physical pages recorded in the byte-state GC table. Single link tables are used to maintain invalid bytes information entries of the same pages. On an onode partial update, the physical page is still marked valid in the page bitmap table. The byte-state GC table records the invalid bytes information entry immediately if the byte-state GC table has free space. If the byte-state GC table is full, then the physical page with the most invalid bytes will be selected for eviction first. After the valid bytes of the evicted page migrate to a clean page, the evicted physical page is invalidated in the page bitmap table and all invalid bytes information entries belonging to the evicted page are removed from the byte-state GC table.

An example of MLGC is depicted in Figure 5. On an onode partial update, the old onode stored at offset 150 in physical page 4 is invalidated. The invalid bytes information <physical page number 4 , offset 150 , length $50>$ is recorded in the byte-state GC table. When an onode partial update on physical page 0 arrives, the byte-state GC table is full and eviction on byte-state GC table is triggered. The physical page with the most invalid bytes, that is, physical page 5 with 200 invalid bytes, is selected for eviction. After moving the remaining valid bytes to physical page 127 , physical page 5 is invalidated in the page bitmap table. All the invalid bytes information entries in physical page 5 are removed from the byte-state GC table.

By adopting two levels of tables, the memory consumption can be reduced to an acceptable level. As we shall show in Section 9, memory consumption of the byte-state GC table is marginal. Thanks to low storage overhead, the byte-state GC table is stored in DRAM for fast access. The byte-state GC table is implemented with link tables and a hash table that maintains the head addresses of invalid bytes information link tables and total invalid bytes of the each physical pages. To quickly find the physical pages with the most invalid bytes for eviction, we also maintain $M$ hash tables (referred as invalid bytes (IB) hash tables). The IB hash table $M-1$ points to the link tables of the physical pages with most invalid bytes. The IB hash table 0 points to the link tables of the 


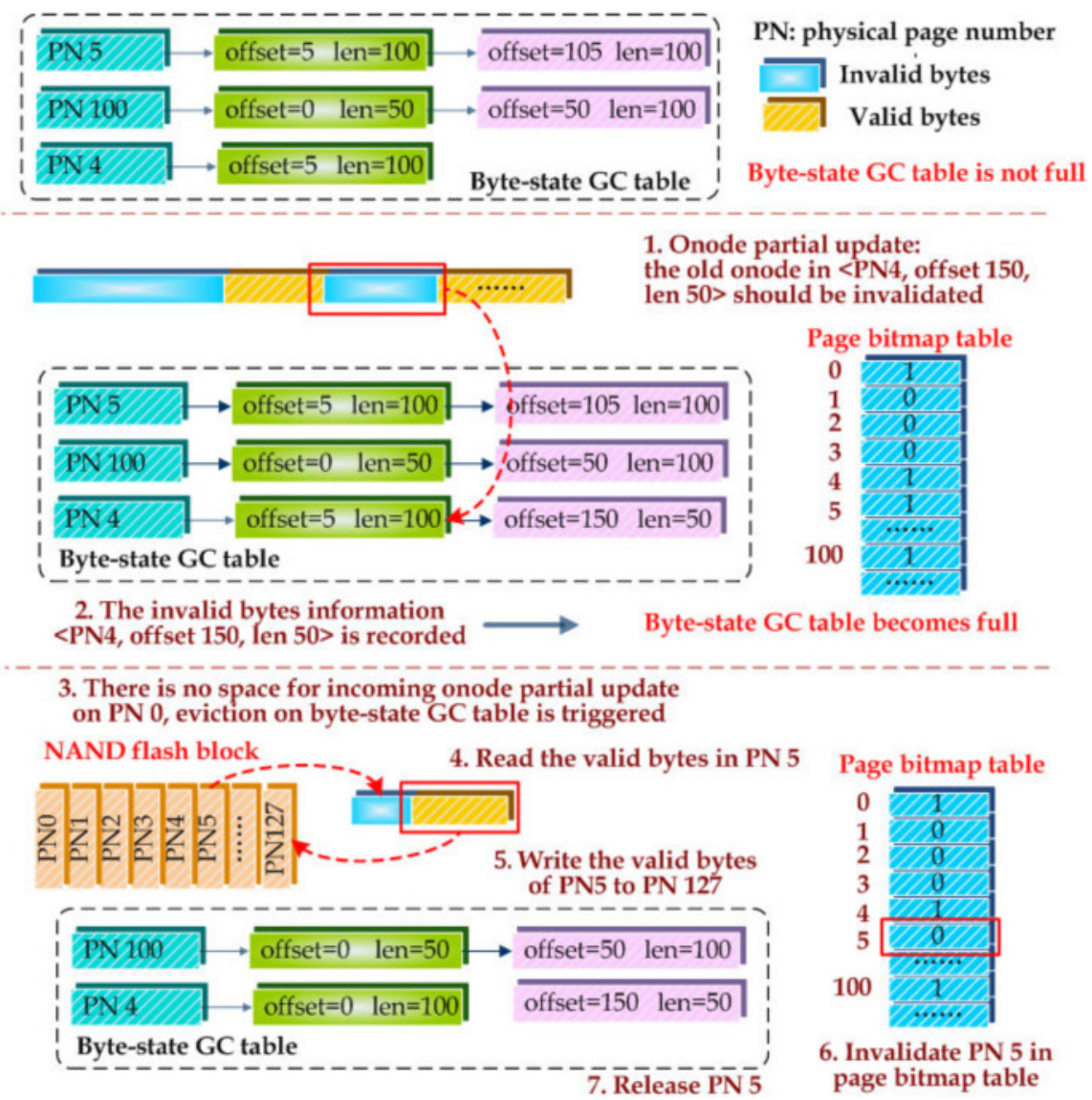

Fig. 5. Multi-level garbage collection (MLGC).

physical pages with fewest invalid bytes. To evict the physical pages with most invalid bytes from the byte-state GC table, we pick the physical pages in the IB hash table $M-1$. Inserting, deleting, and searching for the information of invalid bytes in the byte-state GC table and IB hash tables only involve insertion, deletion, and search operations to hash tables, inserting at the head of the link table and deleting the entire link table. Hence, time complexity of insertion and search operations of byte-state GC table and IB hash tables is $\sim O(1)$, whose performance overhead is negligible.

\section{SEMANTICS-AWARE FLEXIBLE (SAF) DATA LAYOUT}

We propose SAF data layout to reduce cascade update by minimizing separation of the object metadata and object data. As shown in Figure 6, SAF data layout adopts two onode formats: default onode format and extended onode format. Under both onode formats, the entire onode has to be updated as a whole. Like onode in Kang et al. (2011), the onode only stores object attributes and per-object index root node page address under the default onode format. In contrast, the extended onode format allows the per-object index root node or object data to be stored together with attributes in the onode. Selection of onode format for each object is dynamically determined by the size of per-object index and object data. If the total size of attributes and object data of the same 


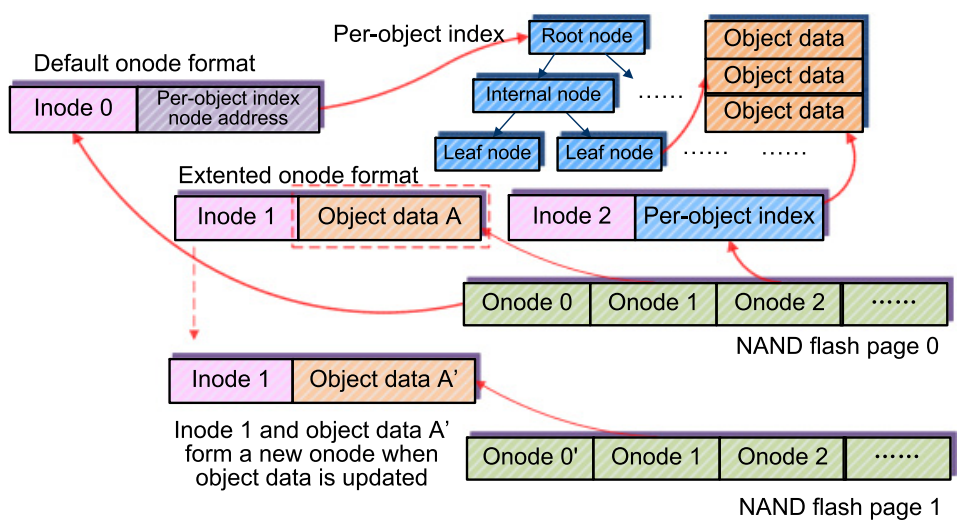

Fig. 6. The SAF data layout.

object is smaller than one physical page, then no per-object index is generated. The object data and attributes are stored in one onode with the extended onode format. We also adopt the extended onode format for objects with the small sized per-object index. If the total size of attributes and the per-object index of an object is smaller than one physical page, then the per-object-index and attributes are stored in one onode. Otherwise, the object will adopt the default onode format and the corresponding onode, per-object index, and object data are stored in different physical pages. Compared with Kang et al. (2011), which invalidates two pages of object metadata on object data update, our SAF design can limit data invalidation to one physical page of object metadata. As such, migration of un-updated data in the invalidated page can be reduced. Adopting the extended onode format can also reduce the overhead of object deletion. When an object is deleted, its onode, per-object index, and data are all invalidated. Grouping object data or per-object index together with attributes in one physical page can effectively reduce invalidated page number the and avoid unnecessary data migration.

Despite effectiveness in mitigating write amplification in the write-intensive workload, adopting the extended onode format may incur unnecessary data migration in the read-intensive workload. When applications read object data through file system, atime in attributes is updated after object data is retrieved. As mentioned in earlier text, once the extended onode format is adopted, object data or per-object index has to migrate together with attributes even though the object data or perobject index is not updated. Hence, for the read-intensive workload, the extended onode format substantially increases unnecessary data migration, directly aggravating write amplification. To avoid the unnecessary data migration, we apply different onode formats to objects with different access patterns. For frequently read and infrequently written objects, we adopt the default onode format to avoid unnecessary data migration. For frequently written and infrequently read objects, we adopt the extended onode format. For frequently written and frequently read (FWFR) objects, we adopt the extended onode format. The reason to use the extended onode format for FWFR objects is that our design deploys selective cache (depicted in Section 7). The selective cache can effectively merge the updates of attributes during read due to the small size of attributes. After merging attributes updates, cascade update is the major overhead for the FWFR objects and the extended onode format can effectively mitigate it. To maximize the efficiency of the SAF data layout, accurate identification of object access patterns is critical. In object-based storage model, accurate identification of object access patterns is easy to realize. We can acquire information of data access patterns based on the file name extension. For example, the files with extension .mp3 


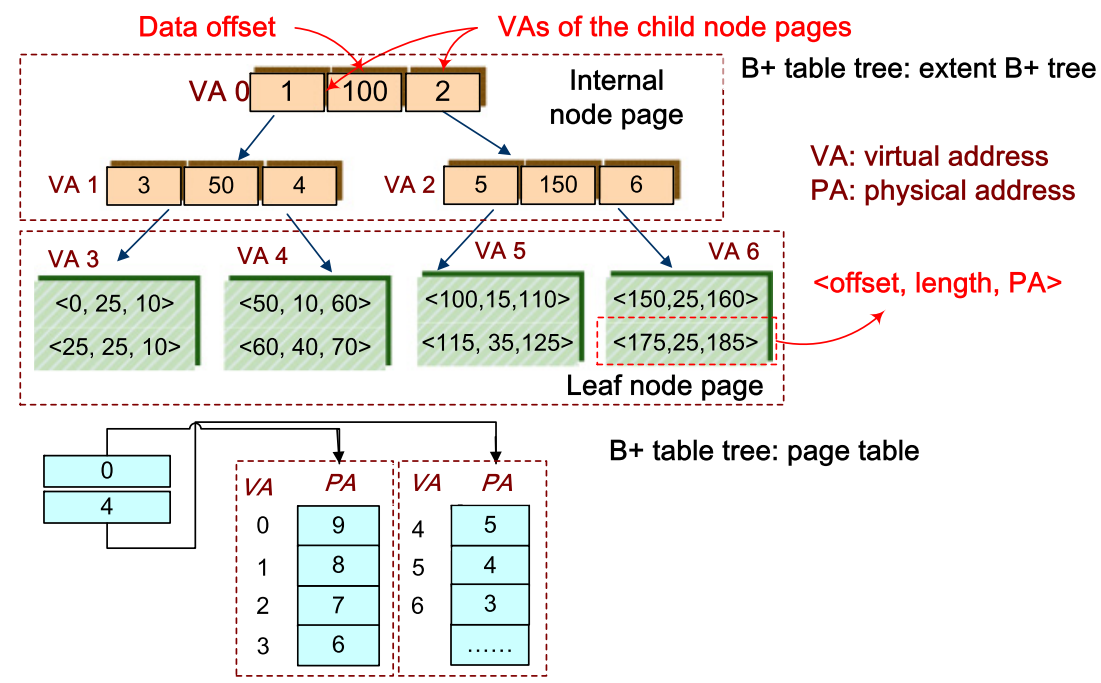

Fig. 7. The $B+$ table tree.

and .jpg, and so on, are frequently read. We can also enable the application to pass an access pattern indication to attributes by modifying APIs of the virtual file system and the object file system.

\section{B+ TABLE TREE}

The $\mathrm{B}+$ table tree is proposed to minimize data migrations incurred by cascading updates. The concept of the $\mathrm{B}+$ table tree is illustrated in Figure 7. The $\mathrm{B}+$ table tree is composed of an extent $\mathrm{B}+$ tree and a page table. The extent $\mathrm{B}+$ tree of $\mathrm{B}+$ table tree has same structure as PA-extent $\mathrm{B}+$ tree. Instead of the physical address $(\mathrm{PA})$, the internal node page in the extent $\mathrm{B}+$ tree of $\mathrm{B}+$ table tree stores the virtual address (VA) of its child node pages. The relationship between VAs and PAs is maintained in a two-level page table. The second level of the page table maintains the relationship between physical addresses and virtual addresses. The second level of the page table are evenly divided into a number of segments. The first level maintains the mapping between the address of each segment and segment indices. The starting virtual address of one segment can be calculated by multiplying the corresponding segment index and the number of virtual addresses in each segment. We only keep the allocated VAs in the page table to reduce memory usage and store the page table in DRAM for fast access. When a child node page migrates to a new page, the new PA is reflected in the page table instead of its parent node page. As such, updates of the internal node pages can be reduced. We also propose to store the VA of the per-object index root node page in the onode. We note that splitting and merging tree nodes may cause update of the root node page, leading to onode update. By recording VA of the per-object index root node page instead of PA in the onode, onode update can be reduced, too.

The insertion and deletion overheads of the $\mathrm{B}+$ table tree are depicted in Table 1 . The overheads are evaluated at the cost of the number of page writes. $H$ and $L$ denote the tree height and the number of splitting nodes, respectively. The overheads of insertion without splitting tree nodes and deletion without merging tree nodes are always 1 , which is only $1 / H$ of the overhead of the PA-extent $\mathrm{B}+$ tree. Figure 8 (a) shows the overhead of insertion without splitting a tree node. Nodes A, B, and C are stored in P1, P2, and P3, respectively. P1, P2, and P3 are mapped to V1, V2, and V3, respectively. It is assumed that the leaf node $\mathrm{C}$ is not full. When a new leaf node entry 
Table 1. Write Overhead of the B+ Table Tree

\begin{tabular}{|c|c|c|}
\hline Operations & $\begin{array}{c}\text { PA-extent } \\
\mathrm{B}+\text { tree }\end{array}$ & $\begin{array}{c}\mathrm{B}+\text { table } \\
\text { tree }\end{array}$ \\
\hline Insertion without splitting tree nodes & $H$ & 1 \\
\hline Insertion with splitting tree nodes & $2 L+1+(H-L)$ & $2 L+1$ \\
\hline Insertion with splitting tree nodes (worst case) & $2 H+1$ & $2 H+1$ \\
\hline Deletion without merging nodes & $H$ & 1 \\
\hline Deletion with merging nodes (worst case) & $H-1$ & $H-1$ \\
\hline
\end{tabular}

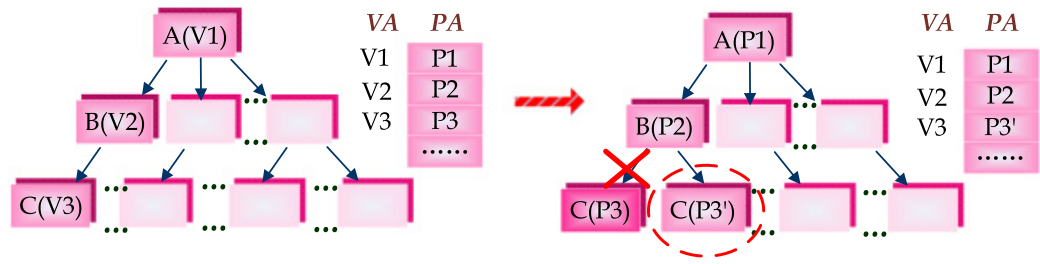

(a) Insertion operation without splitting in B+ table tree.

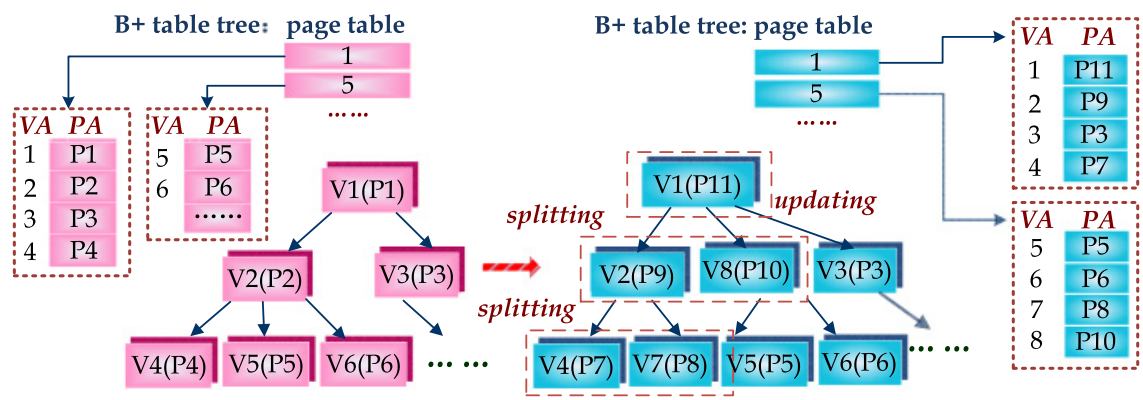

(b) Insertion operation with splitting in B+ table tree.

Fig. 8. Insertion operation of $\mathrm{B}+$ table tree.

is inserted into $\mathrm{C}$, the leaf node page $\mathrm{C}$ is updated and written to $\mathrm{P}^{\prime}$. The $\mathrm{B}+$ table tree only updates the new PA P3' in the page table. Hence, only one per-object index node page migrates. In comparison, three node pages, $\mathrm{A}, \mathrm{B}$, and $\mathrm{C}$, are moved if the PA-extent $\mathrm{B}+$ tree is adopted. When tree node is split during insertion, the splitting node, the newly generated node, and their parent node are updated. Compared with $\mathrm{PA}$-extent $\mathrm{B}+$ tree, the $\mathrm{B}+$ table tree can reduce the page writes by $H$ - L. The worst case happens when the tree splitting cascades to the root node page, as shown in Figure 8(b). In this example, it is assumed that each internal node can have three child nodes at most. Originally, the internal node V2 has three child nodes V4, V5, and V6. Leaf node V4 is full. When a new leaf node entry is inserted to V4, tree node V4 is split. The leaf node V4 splits into two nodes, V4 and V7. The PA of V7 has to be added to the parent node V2. As a result, V2 has to be updated. A total of five page writes are incurred, which is equal to the overhead of the PA-extent $\mathrm{B}+$ tree. Since the worst case occurs much less frequently than any other cases, applying the B+ table tree still significantly reduces the over-write overheads. The way to estimate the deletion overhead is similar to that of insertion.

Besides reducing the overheads of insertion and deletion, the $\mathrm{B}+$ table tree also reduces the overhead of garbage collection. In the PA-extent B+ tree, migration of a child node page involves updating the parent node pages in cascade. Comparatively, in the $\mathrm{B}+$ table tree, migration of a 
child node page is only reflected in the page table. As a result, update and migration of the parent node pages are eliminated.

To minimize write operations to NAND flash memory, we store the page table in the DRAM. Due to the limited size of DRAM, it is crucial to reduce the size of the page table. To reduce the size of the page table, we minimize the number of allocated VAs. Our design only assigns VAs to per-object index node pages instead of both attributes and index blocks as Lee et al. (2015). As such, the size of VA space can be significantly reduced. Adoption of the SAF data layout also contributes to a reduction of the number of VAs. In the SAF data layout, if the total size of the attributes and the per-object index is less than the physical page size, then the attributes and the per-object index are stored in the onode with the extended onode format. Hence, no VA is allocated to the object with the extended onode format and the number of allocated VAs is reduced. We assume that the maximum size of object data is $32 \mathrm{~GB}$ and that the physical page size is $8 \mathrm{~KB}$. The offset, length, and physical page number of the object data recorded in the leaf node page can be represented with $4 \mathrm{~B}, 4 \mathrm{~B}$, and $4 \mathrm{~B}$, respectively. In the worst case, only half of a node page stores indices. Hence, at most $2^{20}$ leaf node pages and $2^{11}$ internal node pages are needed to record the addresses of $2 \mathrm{~TB}$ object data. Each page table entry is represented with $4 \mathrm{~B}$. The maximum size of the page table is only 16MB. Compared with the normal size of DRAM (e.g., several gigabytes), this memory usage is negligible. Due to the small size, the page table can completely reside in DRAM, eliminating NAND flash memory accesses.

Beside reduction of memory consumption, another benefit of only allocating VA to per-object indices is to reduce the storage and performance overheads of the extent $\mathrm{B}+$ tree. With a smaller VA space, VA can be represented by fewer bytes. Hence, the size of a key-value pair (value denotes VA) can be reduced. With the same physical page size, an internal node page can store more keyvalue pairs, leading to a lower tree height and accelerating tree operations. Storing more key-value pairs in one internal node page also lowers the tree re-balancing probability, which further reduces the write accesses to per-object indices in NAND flash memory.

Despite significant reduction of accesses to NAND flash memories, the B+ table tree introduces computation overhead compared with the PA-extent B+ tree. Each time a node page in the NAND flash memory is accessed, the accesses to the page table are required. Hence, the time complexity of insertion, deletion, and search operation to the page table is $\mathrm{O}(H)$. Compared with the write and read latencies of NAND flash memory, the computation overhead is marginal. Hence, the $\mathrm{B}+$ table tree can still significantly improve performance. The proposed B+ table tree can also effectively reduce power consumption. Reduction in write and erase operations naturally decreases dynamic power consumption of NAND flash memory. Similarly, despite introduction of page table lookup, the lookup consumed energy is negligible compared with NAND flash memory operations.

\section{SELECTIVE CACHE}

The $\mathrm{B}+$ table tree can minimize updates of the internal node pages. However, updating object data still generates considerable write accesses to leaf node pages. Besides, tree re-balancing operations such as node splitting and merging also incurs updates of the internal node pages and the onodes. To further reduce updates of object metadata, we propose a selective cache. Previous works proposed some cache replacement policies (Van Riel 2001; Kim and Ahn 2008) to reduce write accesses to NAND flash memory. However, these schemes cause significant memory consumption with the increase of working dataset. Unlike these works, we propose a selective cache to maximize DRAM utilization by fully leveraging object semantics.

First, the selective cache adopts a data-type-dependent caching strategy. The data-typedependent caching strategy is based on the following observation: In the existing object file 
system (Harrosh and Halevy 2009), a page cache is usually used to buffer dirty object data. It is unnecessary to cache the object data in the limited-sized on-device DRAM. Hence, the selective cache only buffers the per-object indices and the updated object attributes. By bypassing largesized object data, memory space is saved for effective caching.

To improve cache write hit rate, we propose a node-dependent cache replacement policy. Our node-dependent cache replacement policy is based on the observation: The per-object index leaf node page is updated in unit of a leaf node entry and only several bytes are written in each update. To achieve effective caching, the selective cache only caches the updated leaf node entries instead of the entire leaf node page. As such, more updates can be buffered and merged in the selective cache. If the cache size exceeds a threshold, then the updated entries of the least recent used (LRU) objects are evicted. The old per-object index leaf node pages are read out and merged with the updated entries. Once a leaf node page is updated, it is immediately committed to NAND flash memory to free memory space. To merge more updates, all the updated internal node pages reside in DRAM until the last updated leaf node pages are committed. Due to tree balancing operations, the new per-object index root node page may be generated, causing onode update. In our design, onode is committed after per-object index internal node pages so updating object attributes and root node pages address can be merged in one onode write. Under such a design, write operations can be significantly reduced.

Caching updated entries instead of the entire node page may lead to increase of read accesses. To minimize read accesses to leaf node pages, we caches the entire page instead of entries when the accesses to an updated leaf node page exceeds a threshold. Usually, internal node pages have lower read miss rate than leaf node pages. Hence, we also buffer internal node pages in the selective cache to facilitate node page search. To fast release memory space for the updated entries, the internal node pages are kept clean during caching. The internal node page update incurred by tree rebalancing is delayed to the time of eviction. The internal node pages of the LRU objects are first released if the selective cache has no space. The internal node pages at higher level have lower probability to be revisited. Hence, the internal node pages at the highest level are released first. As such, cache hit rate can be increased.

A selective cache example is shown in Figure 9. In the selective cache, the updated leaf node entries and clean internal node pages of objects 2 and 3 are buffered. When updating per-object index of object 1, the selective cache is full, and some cache space should be released. The clean internal node pages of objects 2 and 3 are first released. Then, the updated leaf node entries of object 3 are evicted. During eviction, the updated leaf node pages of object 3 are first committed. After all updated leaf node pages are flushed, the updated internal node pages and onode are committed.

The major overhead of the selective cache is memory consumption. During eviction, all the updated internal node pages are buffered in the memory at the same time, potentially increasing memory usage. However, thanks to adoption of the B+ table tree, the updating frequency of the internal node pages is significantly reduced. Hence, the probability to simultaneously buffer many internal node pages is low. The memory consumption of the selective cache is determined by object access patterns and evaluated in Section 9. The selective cache also introduces extra computation overhead. It maintains a hash table to keep track of the object onodes and root node pages of perobject indices stored in the selective cache. The node pages of the per-object indices in the selective cache maintain the structure of binary tree for fast access. The time complexity of accesses to onode in the selective cache is only $\mathrm{O}(1)$. The time to access the node pages of the per-object index in $\mathrm{O}(H)$. However, compared with the reduction of accesses to NAND flash memory, the increase of computation overhead is negligible. 


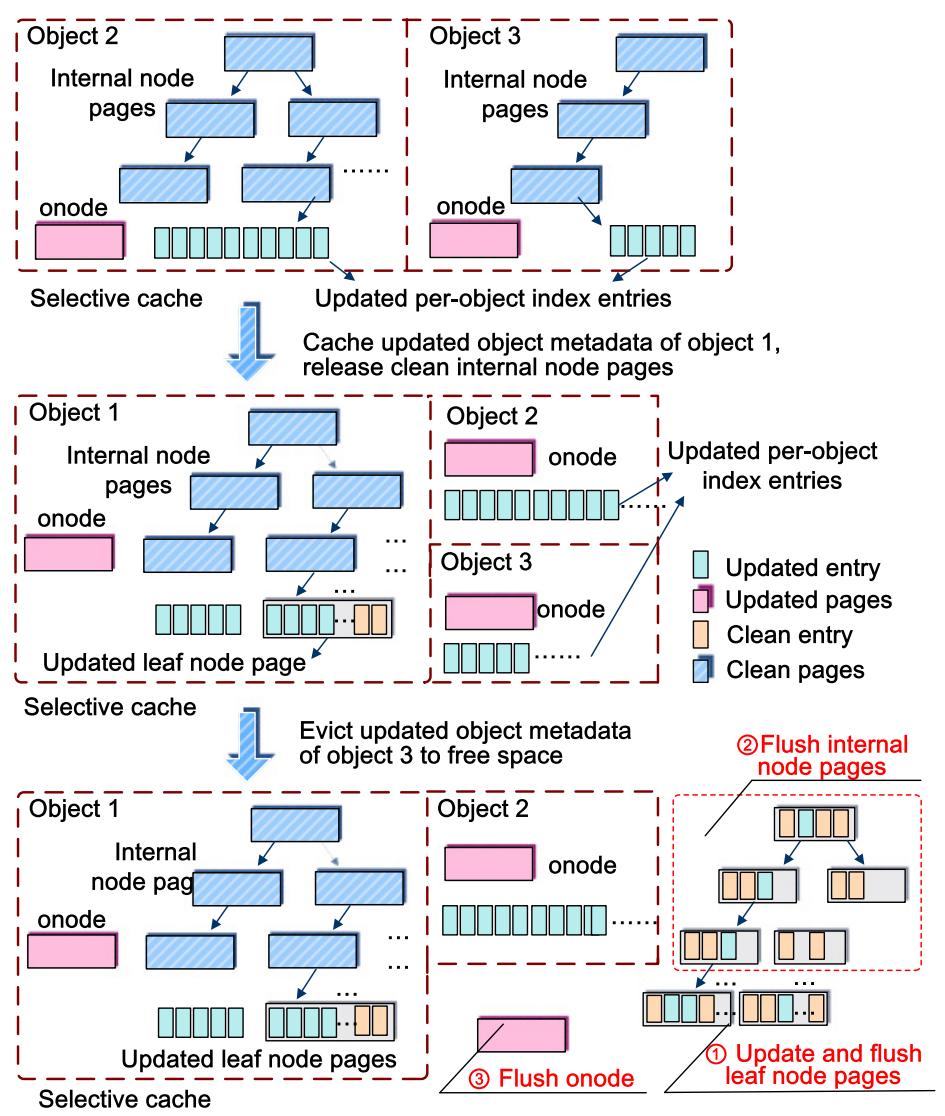

Fig. 9. A selective cache example.

\section{THE POWER FAILURE HANDLING}

The byte-state GC table, the page table of $\mathrm{B}+$ tree, and selective cache are buffered in the on-device DRAM. Power failure will result in loss of tables stored in DRAM and system inconsistency. To restore system consistency and the lost tables, we propose a power failure handling technique. In previous works (Lee et al. 2015), logging and checkpointing are adopted as a consistency restoration technique, incurring significant runtime performance degradation. In comparison, our power failure handling technique can eliminate the runtime overhead by employing page metadata for data recovery and adopting reliable capacitors as backup power. The page metadata includes an object ID, a sequence number (SN), and a VA. The SN represents the sequence of write operations and is automatically incremented after a write operation. To maximize storage efficiency, we adopt different metadata formats for different types of pages, as shown in Figure 10. The onode page metadata contains onode number and onode length. The metadata of per-object index page contains VA. Unlike the storage formats adopted in Lu et al. (2013a), the page metadata and page data both cross the data area and OOB area. This is because the metadata of onode pages is too large to be stored in the $\mathrm{OOB}$ area.

The byte-state GC table can be restored with SNs and object IDs. Only onode partial updates are recorded in the byte-state GC table. During data recovery, the page metadata of the physical pages storing onodes are read out. If more than one physical page stores an onode with the same 


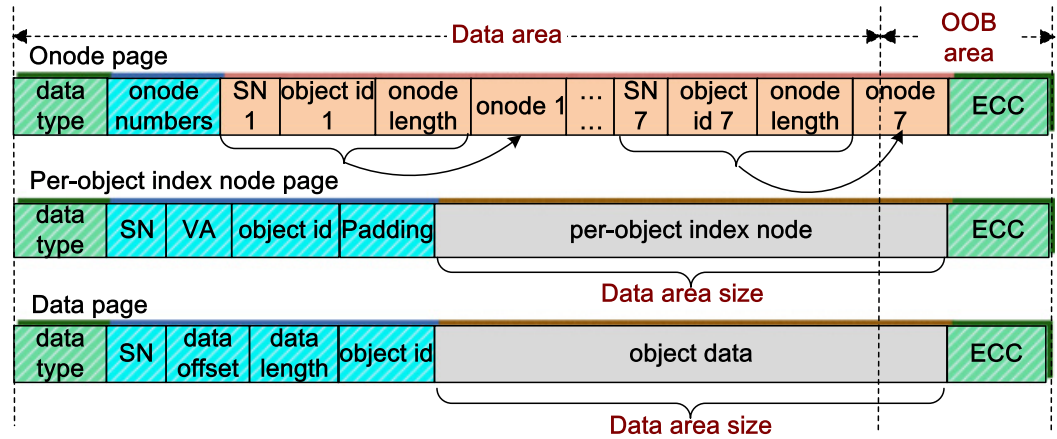

Fig. 10. The format of page metadata.

object ID, then only the onode with the highest $\mathrm{SN}$ is valid. Others are invalidated in the byte-state GC table.

The system consistency can be restored using SNs and VAs through two steps: (1) Per-object indices of the inconsistent objects are rolled back to their own latest consistent state (LCS), and (2) then the physical address of the object data written after the LCS is used to update the per-object indices. Under a LCS, the corresponding per-object index and onode of the updated object data are completely committed. To roll back the per-object indices to the LCS, it is critical to reconstruct a consistent the page table of $\mathrm{B}+$ table tree. To rebuild the page table of $\mathrm{B}+$ table tree, we handle the consistent and inconsistent objects in different ways. For the consistent object, the PAs of the per-object index node pages are inserted to the $\mathrm{B}+$ table tree page table according to the VAs stored in these PAs. If more than one PA has the same VA, then only the PA with the highest SN is inserted. For the inconsistent object, only the per-object index node pages before the LCS can be valid. Hence, the per-object index node pages with SNs higher than the LCS are all invalidated. The PA of a per-object index node page with the highest SN lower than the LCS can be inserted to the $\mathrm{B}+$ table tree page table.

The inconsistent objects are only the objects buffered in the selective cache. The LCS of the inconsistent object can be represented by the $\mathrm{SN}$ of its latest committed onode. To quickly identify the inconsistent objects, the object IDs and SNs of the latest committed onodes of the objects in the selective cache are flushed to NAND flash memory by leveraging backup power on power failure. The cached object attributes are also flushed to NAND flash memory to prevent data loss. Due to small size, flushing these data consumes very low energy. Thus, highly reliable tantalum capacitors can be used as backup power. After being rolled back to the LCS, the object data of these inconsistent objects are read to update the corresponding per-object indices: If the inconsistent object have an object data whose $\mathrm{SN}$ is higher than the LCS, then the PAs of these object data are used to update the per-object index. Also, the object size after updating should be also updated in the object attributes stored in the onode. As such, the object metadata become consistent with the object data.

An example of consistency restoration is shown in Figure 11. The power failure occurs when committing updated per-object index pages of object 1 . The updated per-object index pages are not completely committed: VA1, VA2, VA5, and VA6 are committed while VA0 is still in the selective cache. Hence, object 1 has inconsistent metadata and data. During restoration of the $\mathrm{B}+$ table tree page table, the per-object index of object 1 is rolled back to the LCS, which is SN5: VA1 and VA2 are mapped to PA2 and PA0 whose SNs are lower than SN5; V5 and V6 have SNs higher than SN5 and therefore are invalidated. After restoring the page table, the inconsistent object data stored 


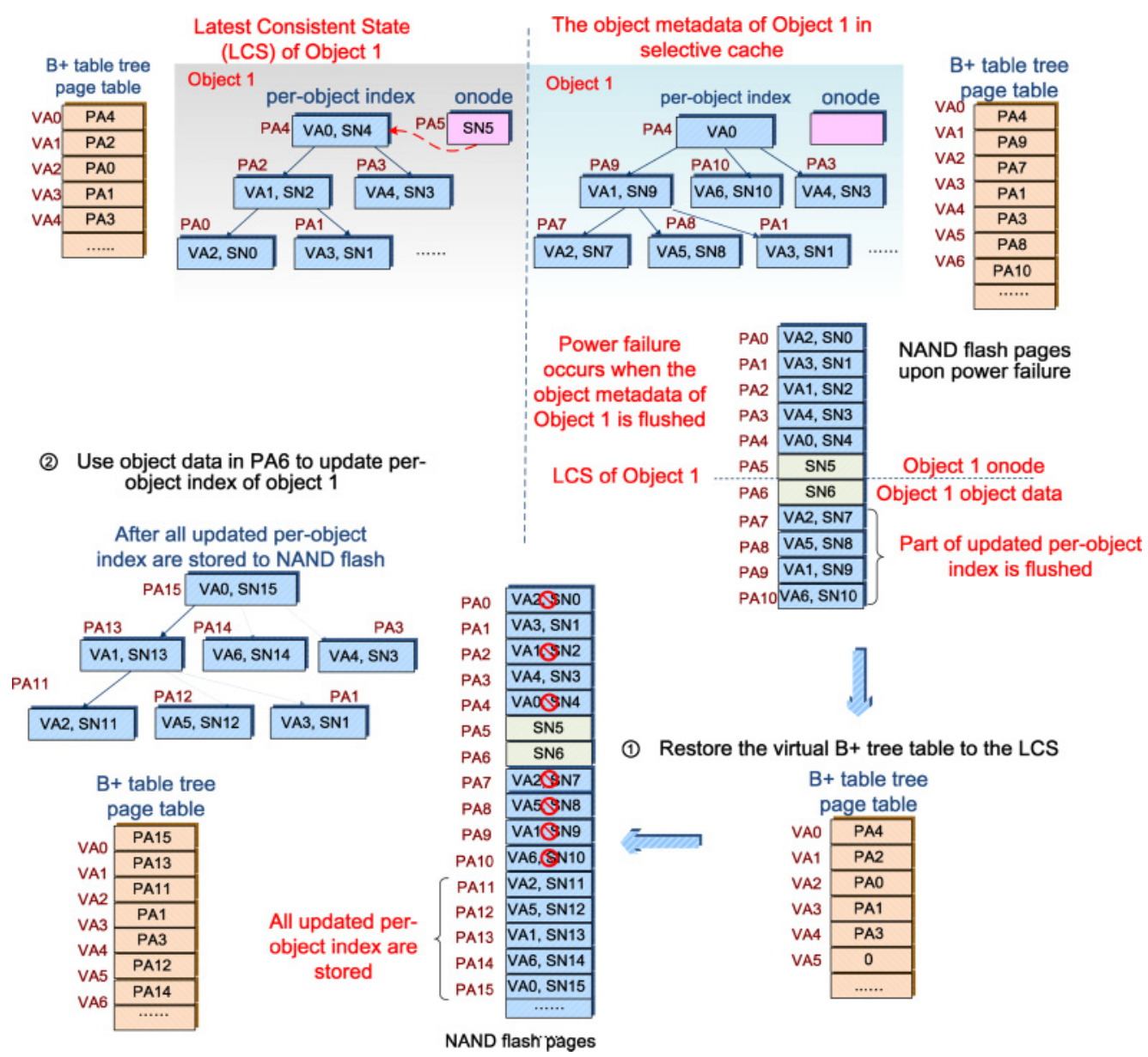

Fig. 11. An example of per-object index recovery.

in PA6 with SN6 is used to update the per-object index. The updated per-object index pages VA0, VA1, VA2, VA5, and VA6 are all flushed to PA15, PA13, PA11, PA12, and PA14, respectively. Finally, object 1 onode with the updated data size and the new per-object index root address is flushed.

\section{EVALUATION}

\subsection{Object-Based Model Prototype}

To evaluate the efficiency of the proposed design, we develop an object-based storage model prototype. The prototype incorporates an object file system, an object storage device (OSD) initiator and an ONFD simulator (Guo et al. 2016b). Here, Exofs in Linux kernel is adopted as the object file system (Harrosh and Halevy 2009). In Exofs, file data and attributes are buffered in the page cache before being flushed to the ONFD simulator. The OSD initiator is an existing Linux kernel library that generates OSD request packets according to SCSI OSD command sets (Nagle et al. 2008). The OSD initiator connects the ONFD simulator via the Gigabit Ethernet. The ONFD simulator is implemented based on an open source software OSC-OSD (Center 2013). Within OSC-OSD, we implement the ONFD architecture shown in Figure 2. The ONFD simulator also emulates the 
Table 2. The Default Parameters of NAND Flash Memory

\begin{tabular}{|c|c|c|c|}
\hline \multirow{2}{*}{ Capacity } & Block size & Block number & Page size \\
\cline { 2 - 4 } & $512 \mathrm{~KB}$ & 8192 & $2 \mathrm{~KB}$ \\
\hline \multirow{2}{*}{ Timing } & Program latency & Read latency & Erase latency \\
\cline { 2 - 4 } & $900 \mu \mathrm{s}$ & $50 \mu \mathrm{s}$ & $1,500 \mu \mathrm{s}$ \\
\hline
\end{tabular}

Table 3. Workload Characteristics

\begin{tabular}{|c|c|c|c|c|c|c|}
\hline Name & Workloads & $\begin{array}{c}\text { Write size } \\
(\mathrm{MB})\end{array}$ & $\begin{array}{c}\text { Write } \\
\text { access (\%) }\end{array}$ & $\begin{array}{c}\text { Write } \\
\text { seq. (\%) }\end{array}$ & $\begin{array}{c}\text { Max file } \\
\text { size (MB) }\end{array}$ & $\begin{array}{c}\text { Avg. write } \\
\text { req. size (KB) }\end{array}$ \\
\hline $\begin{array}{c}\text { DVORAK (Coda) } \\
\text { (University 1994) }\end{array}$ & $\begin{array}{c}\text { Random read to } \\
\text { many small files }\end{array}$ & 602 & 4 & 85 & 67 & 0.5 \\
\hline TPCC & $\begin{array}{c}\text { Random read and } \\
\text { write to large files }\end{array}$ & 7,111 & 24.7 & 19 & 14,290 & 15.7 \\
\hline $\begin{array}{c}\text { IMOVIE (iBench) } \\
\text { (Harter et al. 2012) }\end{array}$ & $\begin{array}{c}\text { Sequential write } \\
\text { to large files }\end{array}$ & 158 & 60.26 & 92 & 66 & 21 \\
\hline $\begin{array}{c}\text { IPHOTO (iBench) } \\
\text { (Harter et al. 2012) }\end{array}$ & $\begin{array}{c}\text { Random write } \\
\text { to large files }\end{array}$ & 5,757 & 90.27 & 36 & 991 & 12 \\
\hline $\begin{array}{c}\text { IPAGE (iBench) } \\
\text { (Harter et al. 2012) }\end{array}$ & $\begin{array}{c}\text { Random write } \\
\text { to small files }\end{array}$ & 179 & 90.81 & 1 & 751 & 0.5 \\
\hline
\end{tabular}

hardware behaviors of NAND flash memory including (1) latencies of program, erase and read operations and (2) multi-channel parallelism.

\subsection{Simulation Setup}

We run the object-based model prototype on a single machine to minimize the impact of the network overhead on the performance evaluation. The machine is equipped with an Intel Quad-Core Xeon 5-2609 v2 (10MB 2GHz) processor and 128GB RAM. The operating system is RHEL 5 with Linux kernel 3.2.67. The configuration of ONFD simulator is set as follows: The device capacity is set to $64 \mathrm{~GB}$ with 16 NAND flash memory dies; the channel number and the chunk size are 16 and $2 \mathrm{MB}$, respectively. The default NAND flash memory parameters are shown in Table 2. A simple garbage collection initiating policy is adopted in this experiments: If the number of clean chunks is lower than a threshold, then the garbage collection is started. Due to large storage space, we set a low clean chunk threshold to quickly initiate garbage collection. The reclaimed chunk is selected in a greedy manner: The chunk with the fewest valid pages is selected for reclamation (Lee et al. 2013).

We evaluate the efficiency of our proposed design under five real-world workloads. DVORAK and TPCC are enterprise applications, and other workloads represent consumer application. Among the five workloads, four workloads are characteristics of either sequential or random writes, which are the major target of our design. We also evaluate the effectiveness of our design in performance improvement under the read-intensive workload, since read operations generate metadata writes. The characteristics of these workload traces are depicted in Table 3. The workload TPCC is generated by Hammerora (GNU 2011) and captured with strace utility (Linux 2016). We write a replay program to generate input to the object-based model prototype from these 


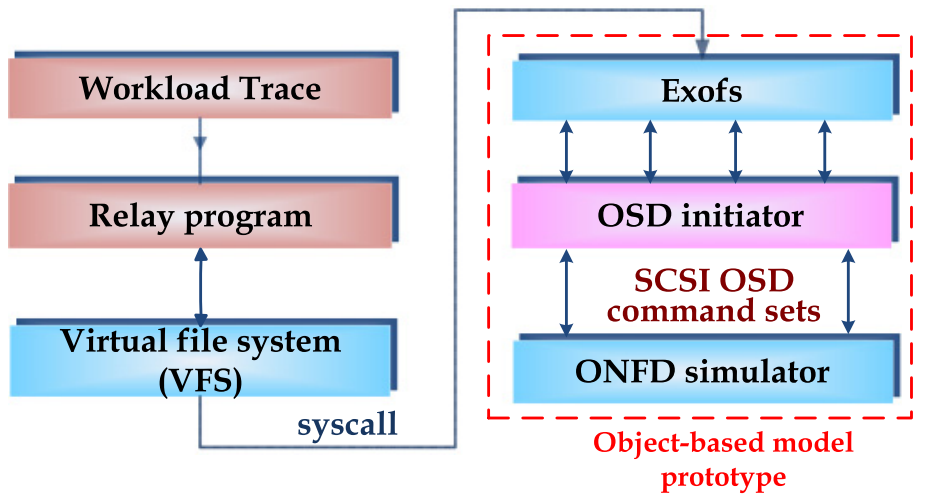

Fig. 12. The object-based storage prototype.
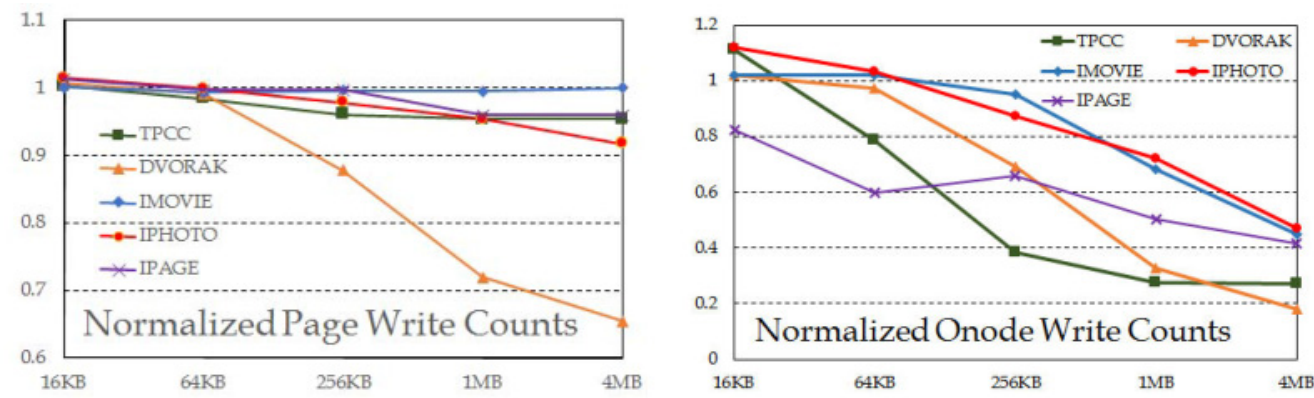

(a) The normalized MLGC page write counts under (b) The normalized MLGC onode write counts under different byte-state GC table sizes. different byte-state GC tables sizes.

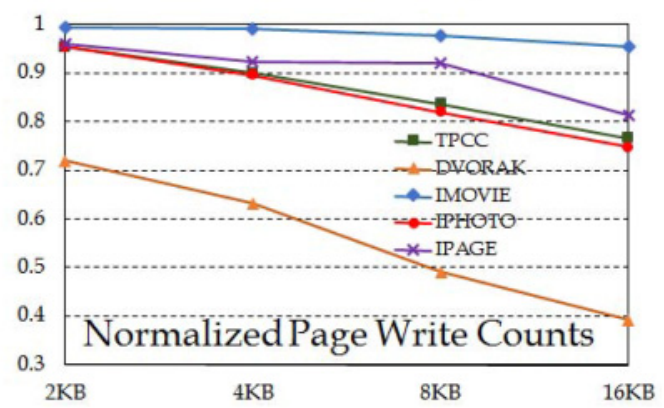

(c) The normalized MLGC page write counts under different physical page sizes.

Fig. 13. The performance of MLGC.

workload traces as shown in Figure 12. We also employ the replay program to measure the I/O response time.

\subsection{Evaluation of MLGC}

We first evaluate the efficiency of MLGC. Figure 13 shows the normalized MLGC write counts under different byte-state GC table sizes. The results are normalized to the write count of the ONFD without MLGC. Compared with the ONFD without MLGC, the page write count under 
a $4 \mathrm{MB}$ byte-state GC table decreases by $11 \%$ on average. The reduction of the page write count comes from the reduction of the onode write count: On average, the onode write count under $4 \mathrm{MB}$ byte-state GC table reduces by $65 \%$ w.r.t. the ONFD without MLGC.

The maximum page write count reduction $(65 \%)$ and the maximum onode write count reduction $(83 \%)$ occur in the read-intensive workload DVORAK. The write count reduction can be explained by the fact that the syscall read invokes atime modification. Frequent read operations at the application layer generate a large amount of object attributes updates and invokes write operations of onodes. Since write accesses to NAND flash memories mainly derives from onode write, reduction on the onode write significantly reduces page writes. MLGC achieves only $1 \%$ page write reduction under the workload IMOVIE. The small page write reduction results from a large amount of sequential writes and a large write request size $(21 \mathrm{~KB})$. A larger write request size results in fewer onode write operations. Hence, the contribution of onode write operations to total page writes becomes less, leading to a smaller page write reduction.

The byte state GC table size is critical to the efficiency of MLGC. Under the workloads TPCC and IPAGE, the page write counts under a $16 \mathrm{~KB}$ byte-state GC table are slightly higher than that without MLGC. The page write increase can be explained by redistribution of reclaimed chunks. MLGC reduces updates of onode pages. Hence, the probability of reclaiming the chunks storing onode is reduced. More chunks storing object data (i.e., object data chunk) are reclaimed. Reclamation of object data chunks invokes updates of the corresponding per-object indices and onodes, leading to write operation increase. Due to the small table size, MLGC cannot group enough amount of onode partial updates. Hence, the data migration increase offsets the onode write reduction by MLGC, leading to page write increase.

When the size of the byte state GC table increases, the page write count is reduced. As shown in Figure 13(a), MLGC with a 256KB byte-state GC table achieves page write count reduction under most workloads. With a relatively large table size, MLGC can group and merge a large mount of onode partial updates. Thus, MLGC can greatly reduce the write operations even though there is a slight increase of reclaimed object data chunks. MLGC with a 1MB byte-state GC table achieves the highest page write count reduction rate under most workloads. This result shows that MLGC can reduce page writes with a marginal storage overhead.

The increase in the size of the physical page is a manufacturing trend (Grupp et al. 2012). Hence, we compare the efficiency of MLGC under different physical page sizes. When increasing the size of the physical page, we decrease the page number per block to keep the block size unchanged. Other system configurations remain the same. In the ONFD with MLGC, a 1MB byte-state GC table is adopted. The normalized MLGC page write counts under different page physical sizes are depicted in Figure 13(c). The write count with MLGC is normalized to the one of the ONFD without MLGC under the same page physical size. Under all workloads, the page write reduction rate increases as the size of the physical page increases. This can be explained by a higher probability that more onode partial updates occur within one page under a larger physical page size. This result demonstrates that MLGC technique is scalable and works better under larger page sizes. In addition, this result also shows phenomenal effectiveness of MLGC with very marginal overhead: Under a $16 \mathrm{~KB}$ page size, a mere $1 \mathrm{MB}$ byte-state GC table can achieve on average $27 \%$ page write count reduction.

\subsection{Evaluation of the B+ Table Tree}

The $\mathrm{B}+$ table tree is evaluated under the default configuration with a $1 \mathrm{MB}$ byte-state GC table. We compare the efficiency of the B+ table tree to the node address table (NAT) used in Lee et al. (2015) and the PA-extent $\mathrm{B}+$ tree. The write count and erase count are normalized against the result of the ONFD with $1 \mathrm{MB}$ MLGC and PA-extent B+ tree. The results in Figure 14 show that the block erase 


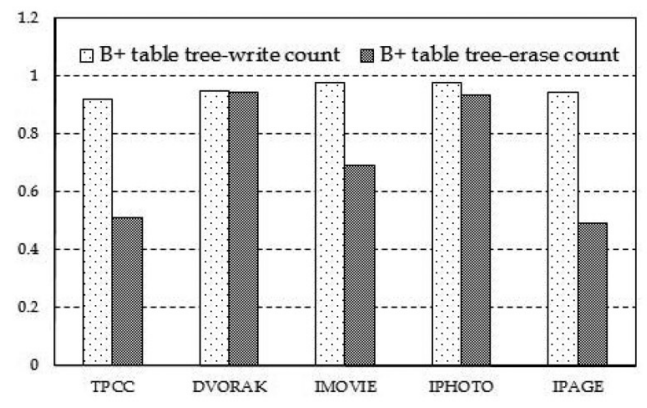

(a) The normalized page write and block erase (b) counts under $\mathrm{B}+$ table tree.

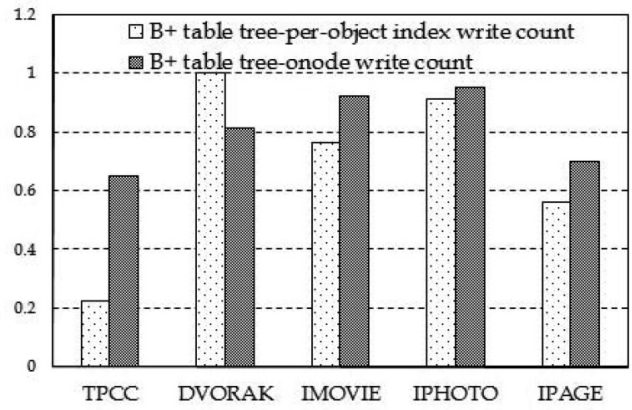

(b) The normalized per-object index and onode write counts under $\mathrm{B}+$ table tree.

Fig. 14. The efficiency of the $B+$ table tree.

count are reduced by $15 \%$ on average compared with NAT. The write reduction can be explained by reduction of the page table incurred write operations. The $\mathrm{B}+$ table tree shows different impacts under different workloads. As depicted in Figure 14(a), the maximum write count reduction of $\mathrm{B}+$ table tree $(9 \%)$ occurs under TPCC. The page write count reduction includes reduction of perobject index writes (77\%) and onode writes (35\%) as shown in Figure 14(b). TPCC is featured by frequent updates of large-sized database tables which have high per-object index trees. With the high extent $\mathrm{B}+$ tree, updating a leaf node page invokes many updates of internal node pages. On the contrary, updates of internal node pages can be effectively reduced in the B+ table tree. Thanks to the decrease in write operations of internal node pages and adoption of VA in onodes, the number of onode updates is also reduced.

In comparison, the page write count is only reduced by $3 \%$ under IMOVIE. This small page write reduction can be explained by the low per-object index tree height. The extent allocation can significantly reduce the per-object index size and tree height under sequential write I/O patterns. There are only a slight page write count reduction (1\%) and onode write count reduction (1\%) under DVORAK. The slight write performance improvement can be explained by the fact that many of the frequently accessed objects have small sizes. These small-sized objects have data and onode stored together without per-object index. Hence, writes of the internal node pages only account for a small portion of the write operations of per-object index. Consequently, write reduction of the $\mathrm{B}+$ table tree has only slight contribution to the overall page write count reduction.

\subsection{Evaluation of Selective Cache}

The efficiency of the selective cache is evaluated under the default system configuration with a $1 \mathrm{MB}$ byte-state $\mathrm{GC}$ table and the $\mathrm{B}+$ table tree. The normalized page write count and the perobject write count under the selective cache against the ONFD only with a $1 \mathrm{MB}$ byte-state GC table and B+ table tree are presented in Figure 15(a) and Figure 15(b), respectively. Compared with the ONFD without the selective cache, the page write count and the per-object write count are reduced by $15 \%$ and $90 \%$ on average under a $2 \mathrm{MB}$ selective cache. The maximum page write count reduction occurs to TPCC: The page write count and per-object index count are reduced by $30 \%$ and $95 \%$, respectively. Results show that the selective cache can effectively reduce per-object index write operations under the workloads with intensive random write accesses. The minimum page write count reduction occurs under IMOVIE: With a $4 \mathrm{MB}$ selective cache, the page write count only decreases by $3 \%$. Nonetheless, $80 \%$ of per-object index write is reduced and a cache hit rate of $95 \%$ is achieved, as shown in Figure 15(c). Under the large-sized sequential write I/O pattern, 

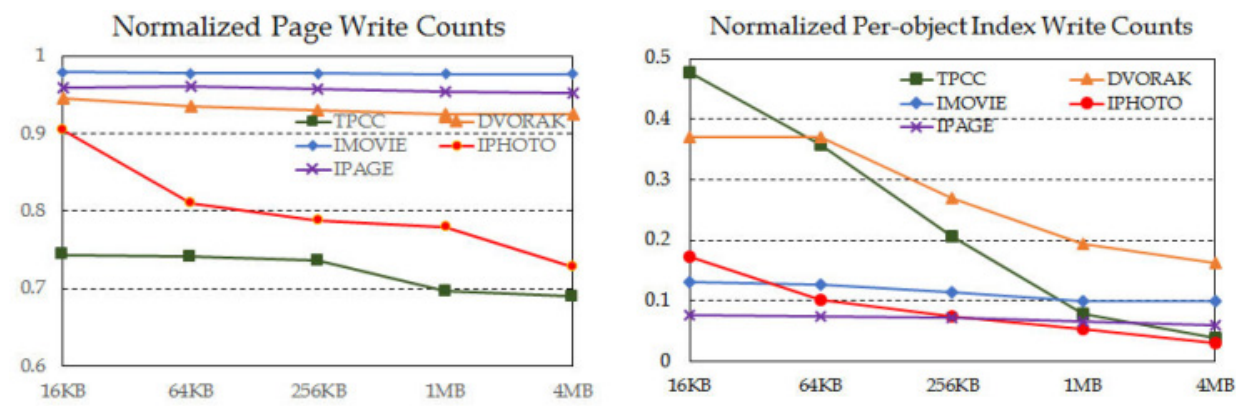

(a) The normalized page write counts under (b) The normalized per-object index write counts different selective cache sizes. under different selective cache sizes.
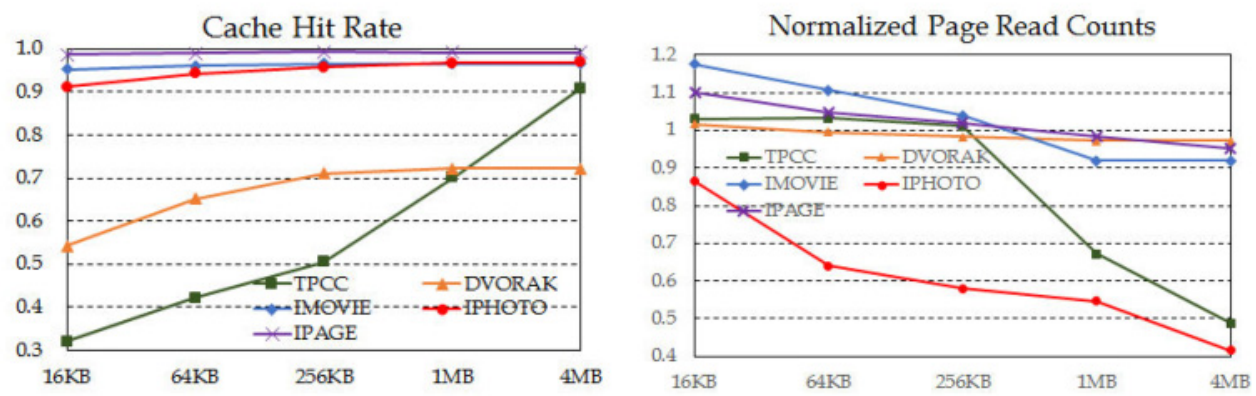

(c) The cache hit rates under different selective (d) The normalized page read count under the cache sizes. selective cache.

Fig. 15. The efficiency of the selective cache.

the per-object index write operations only account for a small portion of the overall page writes. Hence, a large per-object index reduction only achieve a small reduction of the overall page write count. A $4 \mathrm{MB}$ selective cache reduces the page write count by $5 \%$ but decreases per-object index count by $92 \%$ under IPAGE. This relatively small page write count reduction can be explained by redistribution of the reclaimed chunk type. With the significant reduction of write accesses to the per-object indices, more chunks storing object data are reclaimed. The increase of object data write operations partially offsets the performance gain of the selective cache. We believe our selective cache can show more benefits by improving the garbage collection algorithm. For example, both data type and valid page number can be utilized together to select the reclaimed chunks. The page write count reduction is $7.5 \%$ under DVORAK. There is also a large per-object index write reduction $(85 \%)$ but only a slight page write reduction $(7 \%)$. This is because a large portion of write operations are performed to the small files without per-object index.

The effects of different selective cache sizes are evaluated. Under IMOVIE and IPAGE, the cache hit rate reaches $95 \%$ with a $16 \mathrm{~KB}$ selective cache. The high cache hit rate can be explained by a small write working dataset. The cache hit rate of the $16 \mathrm{~KB}$ selective cache is relatively low under TPCC due to random accesses to the database tables. The cache hit rate increases to $95 \%$ when the selective cache size reaches $4 \mathrm{MB}$. Under a $1 \mathrm{MB}$ selective cache, the per-object index write count is reduced by $86 \%$ and the cache hit rate reaches $87 \%$ on average. These results demonstrate that selective cache can effectively reduce the per-object index writes with only marginal memory usage. We also show the impact of the selective cache on the read overhead in Figure 15(d). The page read count under the selective cache is normalized against the ONFD with a 1MB byte-state 


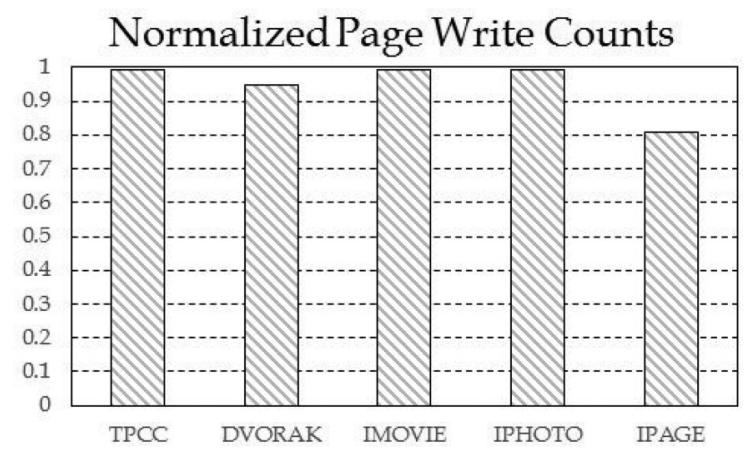

Fig. 16. The normalized page write count under SAF data layout.

GC table and B+ table tree. Thanks to the node-dependent cache replacement policy, the page read count is reduced by $19 \%$ on average under a $1 \mathrm{MB}$ selective cache. The maximum page read count reduction occurs to IPHOTO and TPCC. This is because the operations to the per-object indices are frequently performed to several large files. Hence, the cache read hit rate could be relatively high and therefore more read count reduction is achieved.

\subsection{Evaluation of SAF Data Layout}

We also evaluate the efficiency of the proposed SAF data layout. Figure 16 lists the normalized page write count of ONFD with SAF data layout against a plain ONFD. Compared with the plain ONFD, the page write count of the ONFD with SAF data layout is reduced by $6 \%$ on average. The maximum page write count reduction occurs to IPAGE due to frequent updates of small files. The SAF barely has write performance improvement under TPCC and IMOVIE due to frequent write operations to large files.

\subsection{The Overall Performance Improvement}

Finally, we compare the overall average I/O response time, page write count, and erase write count of our proposed design with OBFS (Kang et al. 2011) and YAFFS2 (Ltd. 2012) under the five workloads. Our design adopts the default system configuration with a $1 \mathrm{MB}$ byte-state GC table, a B+ tree table, and a $1 \mathrm{MB}$ selective cache. We implement OBFS and the storage management functionality of YAFFS in the ONFD simulator. The performance evaluation results of our proposed design are depicted in Figure 17. In these figures, RWA denotes our proposed design. The average response time in Figure 17(a) is the clock time of the ONFD to respond to a request and shown with a linear scale. Compared with YAFFS2 and OBFS, our design reduces the average response time by $28 \%$ and $30 \%$, respectively. Even under the read-intensive workload DVORAK, there is $20 \%$ response time reduction due to significant onode write reduction. Page write count is shown with a log scale in Figure 17(b). Compared with YAFFS2 and OBFS, our design reduces the page write count by $19 \%$ and $25 \%$, respectively. TPCC has the maximum page reduction $(20 \%)$ against OBFS because of a large per-object index write reduction. Since the P/E cycle count represents the endurance of NAND flash memory, reduction of the block erase count can be used to evaluate the endurance improvement. The block erase count in Figure 14(b) shows that our design improves the system endurance by $76 \%$ on average, compared with OBFS. Similar to the page write reduction, the maximum reduction of the block erase count occurs to the TPCC and IPAGE workload, thanks to significant reduction of page writes. 

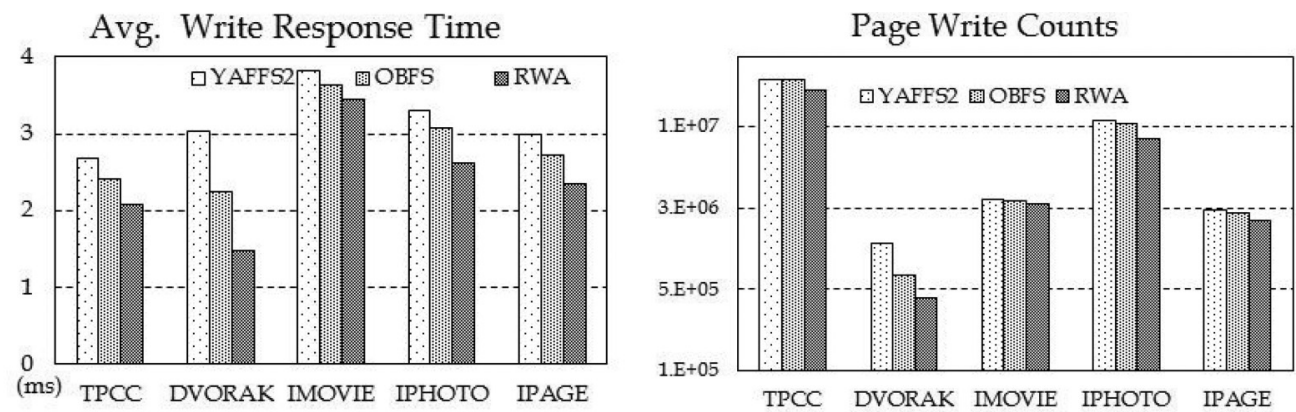

(a) The average write response time of the pro- (b) The page write counts of the proposed design. posed design.

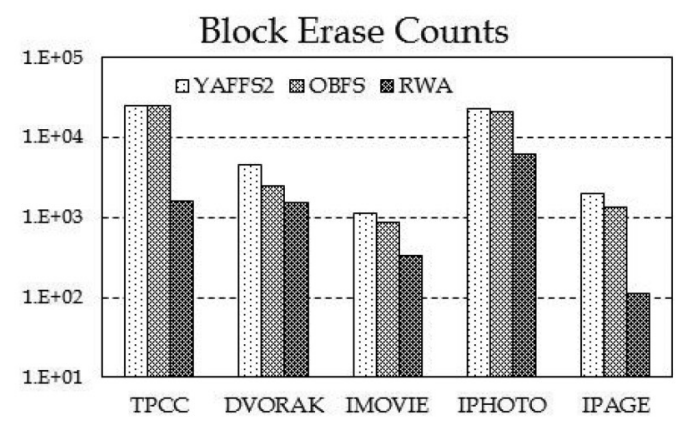

(c) The block erase counts of the proposed design.

Fig. 17. The overall efficiency of our design.

Since memory consumption is the major overhead, we summarize scaling of memory consumption of our proposed design. The memory consumption of the proposed design is associated with the object access patterns, the working data sets and the number of objects stored in the ONFD. The workload is a major factor determining the optimal memory size of our design. The memory consumption of MLGC and selective cache increases with the increase of the working data set size and the decrease of temporal locality. The memory consumption of B+ table tree increases with the increase of the number of objects and random write accesses. Increasing the size of the ONFD could increase the number of the stored objects. As a result, the memory consumption will increase due to the increase in the size of the page table of the $\mathrm{B}+$ table tree.

\section{RELATED WORKS}

Several works have been proposed to reduce partial page update and cascading update induced data migration. Sun et al. (2013) proposed to merge partial page updates using phase change memory, a byte-addressable emerging non-volatile memory. Min et al. (2017) proposed to adopt emerging non-volatile memory STT-RAM to improve the performance of object-based NAND flash device. However, these new types of memories are either expensive or technically unmature. $\mathrm{Lu}$ et al. (2013a) identifies object data induced partial page update under the byte-unit access interface. To mitigate the partial page update, object data updates are compacted in diff-pages before they are merged with the un-updated object data. However, this scheme is not applicable to onode partial update. 
Lee et al. (2015) proposed a node address table to reduce the cost of cascading updates in indirect blocks. However, the introduced node address table is stored in NAND flash memory, causing extra write operations. Similarly to Lee et al. (2015), our proposed B+ table tree adopts a page table to reduce cascading updates. Unlike Lee et al. (2015), we adopt several techniques to reduce the size of the page table of our $\mathrm{B}+$ table tree. As such, the page table can be completely stored in the DRAM, and the related write operations are eliminated. In addition, different from Lee et al. (2015), our work also handles cascading update between different types of object metadata: Our $\mathrm{B}+$ table tree together with the SAF data layout can minimize per-object index root node incurred cascade update by either using VA or directly storing per-object index in the onode. In contrast, Lee et al. (2015) is unaware of cascading update between different types of object metadata. Kang et al. (2007) proposed $\mu$ tree to mitigate cascading update of the $\mathrm{B}+$ tree. The $\mu$ tree realizes write operation reduction by compacting the most recently updated nodes in one page. However, due to the reduced node size, $\mu$ tree increases internal fragmentation. The search overhead of $\mu$ tree also increases due to increase of the tree height. On et al. (2009) proposed to delay and merge updates of the B+ tree using a cache in main memory. However, this lazy update scheme significantly increases main memory usage. Compared with the previous works, our proposed design can more effectively mitigate the write amplification with very minimum memory usage. Lee et al. (2013) proposed object-aware data placement and hot/cold data separation techniques in ONFD to reduce write and deletion overhead. Our proposed design can work together with Lee et al. (2013) to further improve write performance.

This work is an extension of the research by Guo et al. (2016a). Compared with Guo et al. (2016a), we present more details in the motivation of our work in terms of SAF and cascade update in this article. In addition to more quantitative overhead analysis of the proposed schemes, we also provide a detailed and thorough description of the proposed technique SAF data layout and include experimental results to evaluate its efficiency.

\section{CONCLUSION}

In an ONFD, onode partial update and cascading update are identified as two causes of write amplification. To reduce the unnecessary write incurred by onode partial update, MLGC technique is first proposed in this work. MLGC groups invalid data induced by more than one onode partial update and moves the remaining valid data at one time, and, yhereby, migration of unnecessary data can be reduced. The SAF data layout, $\mathrm{B}+$ table tree, and selective cache are then proposed to reduce cascading update of object metadata. Through grouping attributes and per-object index or data of the same object in the same page based on the data access patterns, SAF can reduce the amount of unnecessary data migration. B+ table tree replaces frequently updated physical addresses with unchanged virtual addresses and transfers the address update to a DRAM-resident page table from the internal node pages. As such, updates of internal node pages of per-object indices can effectively reduced. The selective cache adopts a data-type-dependent caching strategy and a node-dependent cache replacement policy to minimize per-object index updates. Our experimental results show that, compared with the best previous work, our proposed design can reduce page write count by $20 \%$ with $76 \%$ system lifetime extension.

\section{REFERENCES}

Ohio Supercomputing Center. 2013. OSC software OSD implementation. Retrieved from https://github.uconn.edu/SNSL/ osc-osd/tree/master.

Vijay Chidambaram, Tushar Sharma, Andrea C. Arpaci-Dusseau, and Remzi H. Arpaci-Dusseau. 2012. Consistency without ordering. In Proceedings of the 10th USENIX Conference on File and Storage Technologies (FAST'12). 9.

GNU. 2011. Hammerora: the open source oracle load test tool. Retrieved from https://sourceforge.net/projects/hammerora/. 
Laura M. Grupp, John D. Davis, and Steven Swanson. 2012. The bleak future of NAND flash memory. In Proceedings of the 10th USENIX Conference on File and Storage Technologies (FAST'12). 2-2.

Jie Guo, Chuhan Min, Tao Cai, and Yiran Chen. 2016a. A design to reduce write amplification in object-based NAND flash devices. In Proceedings of the International Conference on Hardware/Software Codesign and System Synthesis (CODES+ ISSS'16). 1-10.

Jie Guo, Chuhan Min, Tao Cai, Hai Li, and Yiran Chen. 2016b. Objnandsim: Object-based NAND flash device simulator. In Proceedings of the 5th Non-Volatile Memory Systems and Applications Symposium (NVMSA'16). 1-6.

Jie Guo, Danghui Wang, Zili Shao, and Yiran Chen. 2017. Data-pattern-aware error prevention technique to improve system reliability. IEEE Trans. VLSI Syst. 25, 4 (2017), 1433-1443.

Jie Guo, Wujie Wen, Jingtong Hu, Danghui Wang, Hai Li, and Yiran Chen. 2015. Flexlevel: A novel nand flash storage system design for ldpc latency reduction. In Proceedings of the 52nd ACM/EDAC/IEEE Design Automation Conference (DAC'15). 1-6.

Jie Guo, Wujie Wen, Yaojun Zhang Li, Sicheng Li, Hai Li, and Yiran Chen. 2013a. DA-RAID-5: A disturb aware data protection technique for NAND flash storage systems. In Proceedings of the Design, Automation \& Test in Europe Conference \& Exhibition (DATE'13). 380-385.

Jie Guo, Jun Yang, Youtao Zhang, and Yiran Chen. 2013b. Low cost power failure protection for MLC NAND flash storage systems with PRAM/DRAM hybrid buffer. In Proceedings of the Design, Automation \& Test in Europe Conference \& Exhibition (DATE'13). IEEE, 859-864.

Aayush Gupta, Youngjae Kim, and Bhuvan Urgaonkar. 2008. DFTL: A flash translation layer employing demand-based selective caching of page-level address mappings. In Proceedings of the 14th International Conference on Architectural Support for Programming Languages and Operating Systems (ASPLOS'08), Vol. 44.

B. Harrosh and B. Halevy. 2009. The Linux Exofs Object-based pNFS Metadata Server.

Tyler Harter, Chris Dragga, Michael Vaughn, Andrea C. Arpaci-Dusseau, and Remzi H. Arpaci-Dusseau. 2012. A file is not a file: Understanding the I/O behavior of apple desktop applications. ACM Trans. Comput. Syst. 30, 3 (2012), 10.

Jen-Wei Hsieh, Tei-Wei Kuo, and Li-Pin Chang. 2006. Efficient identification of hot data for flash memory storage systems. ACM Trans. Stor. 2, 1 (2006), 22-40.

Xiao-Yu Hu, Evangelos Eleftheriou, Robert Haas, Ilias Iliadis, and Roman Pletka. 2009. Write amplification analysis in flash-based solid state drives. In Proceedings of the ACM International System and Storage Conference (SYSTOR'09). 10.

Dongwon Kang, Dawoon Jung, Jeong-Uk Kang, and Jin-Soo Kim. 2007. $\mu$-tree: An ordered index structure for NAND flash memory. In Proceedings of the 7th ACM \& IEEE International Conference on Embedded Software (ESOFT'07). 144-153.

Yangwook Kang, Jingpei Yang, and Ethan L. Miller. 2011. Object-based SCM: An efficient interface for storage class memories. In Proceedings of the 27th IEEE Symposium on Mass Storage Systems and Technologies (MSST'11). 1-12.

Hyojun Kim and Seongjun Ahn. 2008. BPLRU: A buffer management scheme for improving random writes in flash storage. In Proceedings of the 6th USENIX Conference on File and Storage Technologies (FAST'08), Vol. 8. 1-14.

Changman Lee, Dongho Sim, Jooyoung Hwang, and Sangyeun Cho. 2015. F2FS: A new file system for flash storage. In Proceedings of the 13th USENIX Conference on File and Storage Technologies (FAST'15). 273-286.

Sungjin Lee, Dongkun Shin, Young-Jin Kim, and Jihong Kim. 2008. LAST: Locality-aware sector translation for NAND flash memory-based storage systems. ACM SIGOPS Operat. Syst. Rev. 42, 6 (2008), 36-42.

Yong-Goo Lee, Dawoon Jung, Dongwon Kang, and Jin-Soo Kim. 2008. $\mu$-FTL: A memory-efficient flash translation layer supporting multiple mapping granularities. In Proceedings of the 8th ACM International Conference on Embedded Software (ESOFT'08). 21-30.

Young-Sik Lee, Sang-Hoon Kim, Jin-Soo Kim, Jaesoo Lee, Chanik Park, and Seungryoul Maeng. 2013. OSSD: A case for object-based solid state drives. In Proceedings of the 29th IEEE Symposium on Mass Storage Systems and Technologies (MSST'13). 1-13.

Linux. 2016. Strace-trace system calls and signals. Retrieved from http://man7.org/linux/man-pages/man1/strace.1.html. Aleph One Ltd. 2012. Yaffs A Flash File System for Embedded Use. Retrieved from http://www.yaffs.net/.

Youyou Lu, Jiwu Shu, and Weimin Zheng. 2013a. Extending the lifetime of flash-based storage through reducing write amplification from file systems. In Proceedings of the 11th USENIX Conference on File and Storage Technologies (FAST'13) 257-270.

Youyou Lu, Jiwu Shu, and Weimin Zheng. 2013b. Extending the lifetime of flash-based storage through reducing write amplification from file systems. In Proceedings of the 11th USENIX Conference on File and Storage Technologies (FAST'13). 257-270.

Chuhan Min, Jie Guo, Hai Li, and Yiran Chen. 2017. Extending the lifetime of object-based NAND flash device with STT-RAM/DRAM hybrid buffer. In Proceedings of the 22nd Asia and South Pacific Design Automation Conference (ASPDAC'17). 764-769.

David Nagle et al. 2008. The ANSI T10 object-based storage standard and current implementations. IBM f. Res. Dev. 52, 4.5 (2008), 401-411. 
Sai Tung On, Haibo Hu, Yu Li, and Jianliang Xu. 2009. Lazy-update b+-tree for flash devices. In Proceedings of the 10th International Conference on Mobile Data Management: Systems, Services and Middleware. 323-328.

Abhishek Rajimwale, Vijayan Prabhakaran, and John D. Davis. 2009. Block management in solid-state devices. In Proceedings of the USENIX Annual Technical Conference (ATC'09).

Mendel Rosenblum and John K. Ousterhout. 1992. The design and implementation of a log-structured file system. ACM Trans. Comput. Syst. 10, 1 (1992), 26-52.

Chao Sun et al. 2013. SCM capacity and NAND over-provisioning requirements for SCM/NAND flash hybrid enterprise SSD. In Proceedings of the International Memory Workshop (IMW'13). 64-67.

Carnegie Mellon University. 1994. Coda Project Traces and DFSTrace. Retrieved from http://coda.cs.cmu.edu/DFSTrace/.

Rik Van Riel. 2001. Page replacement in linux 2.4 memory management. In Proceedings of the USENIX ATC FREENIX Track. 165-172.

Danghui Wang, Jie Guo, Kai Bu, and Yiran Chen. 2014a. Reduction of data prevention cost and improvement of reliability in MLC NAND flash storage system. In Proceedings of the International Conference on Computing, Networking and Communications (ICNC'14). 259-263.

Wei Wang, Youyou Lu, and Jiwu Shu. 2014b. p-oftl: An object-based semantic-aware parallel flash translation layer. In Proceedings of the Conference on Design, Automation \& Test in Europe (DATE'14). 157.

Jung Yoon and Gary Tressler. 2012. Advanced flash technology status, scaling trends and implications to enterprise SSD technology enablement. In Proceedings of the Flash Memory Summit.

Kai Zhao, Wenzhe Zhao, Hongbin Sun, Xiaodong Zhang, Nanning Zheng, and Tong Zhang. 2013. LDPC-in-SSD: Making advanced error correction codes work effectively in solid state drives. In Proceedings of the 11th USENIX Conference on File and Storage Technologies (FAST'13). 243-256.

Received December 2016; revised May 2017; accepted May 2017 